\title{
Idéaux stables dans certains anneaux différentiels de formes quasi-modulaires de Hilbert ${ }^{1}$.
}

\author{
Federico Pellarin ${ }^{2}$
}

English abstract. In [9] Nesterenko proved, among other results, the algebraic independence over $\mathbb{Q}$ of the numbers $\pi, e^{\pi}, \Gamma(1 / 4)$. A very important feature of his proof is a multiplicity estimate for quasi-modular forms associated to $\mathbf{S L}_{2}(\mathbb{Z})$ which involves profound differential properties of certain non-linear differential systems.

The aim of this article is to begin the study of the analogous properties for Hilbert modular and quasi-modular forms, especially those which are associated with the number field $\mathbb{Q}(\sqrt{5})$. We show that the differential structure of these functions has several analogies with the differential structure of the quasi-modular forms associated to $\mathbf{S L}_{2}(\mathbb{Z})$.

\section{Introduction.}

Soit $\mathcal{A}$ un anneau commutatif, et $\mathcal{D}$ un ensemble de dérivations sur $\mathcal{A}$. Un idéal $\mathcal{I}$ de $\mathcal{A}$ est stable pour $\mathcal{D}$ (ou $\mathcal{D}$-stable, ou encore, stable si la référence à $\mathcal{D}$ est claire) si pour tout $D \in \mathcal{D}$ on a

$$
D \mathcal{I}:=\{D x, \text { avec } x \in \mathcal{I}\} \subset \mathcal{I} .
$$

Définition. On dit que l'anneau différentiel $(\mathcal{A}, \mathcal{D})$ satisfait la propriété de Ramanujan, s'il existe un élément non nul $\kappa \in \mathcal{A}$ tel que tout idéal premier non nul $\mathcal{I}$ de $\mathcal{A}$ qui est $\mathcal{D}$-stable contient $\kappa$.

Soit $q$ un nombre complexe tel que $|q|<1$, soient $E_{2}(q), E_{4}(q), E_{6}(q)$ les séries de Fourier des séries d'Eisenstein classiques de poids 2,4,6 $\left(^{3}\right)$ et posons $D=q(d / d q)$. On sait alors que $D$ induit une dérivation sur $\mathcal{R}=$ $\mathbb{C}\left[E_{2}, E_{4}, E_{6}\right]$. On peut montrer que l'anneau différentiel $(\mathcal{R}, D)$ satisfait la

${ }^{1}$ AMS Classification : 11F41, 47F05, 35G05. Keywords : Hilbert modular and quasimodular forms, Rankin-Cohen operators, differential ideals, multiplicity estimates.

${ }^{2}$ Laboratoire LMNO, Université de Caen, BP 5186, 14032 Caen Cedex, France.

${ }^{3}$ Notées respectivement $3 \pi^{-2} G_{1}$ p. 156 et $E_{2}, E_{3}$ p. 151 de [15]. 
propriété de Ramanujan : tout idéal premier non nul $D$-stable de $\mathcal{R}$ contient $\Delta=E_{4}^{3}-E_{6}^{2}$.

En fait, aussi l'anneau $\mathcal{R}[q]$ est un anneau différentiel, et on peut démontrer qu'il satisfait la propriété de Ramanujan (voir [12]).

La propriété de Ramanujan est indispensable dans les estimations de multiplicité. Par exemple, Nesterenko a utilisé une propriété plus faible que la propriété de Ramanujan pour $(\mathcal{R}[q], D)$ pour démontrer ce qui suit (cf. [9] et [10], chapitres 3,10$)$.

Théorème $1 \mathrm{Il}$ existe une constante explicite $c>0$ telle que pour tout polynôme $P \in \mathcal{R}$ de degré au plus $N$, on ait :

$$
\operatorname{ord}_{q=0} P \leq c N^{4}
$$

Quant'aux formes modulaires de Hilbert de deux (ou de plusieurs variables complexes), ce serait évidemment très intéressant d'établir des estimations de multiplicité généralisant le théorème 1 pour ces fonctions; avec ce texte, nous voudrions inaugurer cette étude.

Une première motivation de ce texte est d'introduire une notion de forme quasi-modulaire de Hilbert généralisant celle de [8]; elle ne semble pas, à présent, apparaître dans la literature.

Soit $K$ un corps de nombres totalement réel de degré $n$ sur $\mathbb{Q}$, soit $\Gamma_{K}$ le groupe modulaire de Hilbert associé (cf. [5], p. 5). Nous allons définir, au paragraphe 2, un anneau $\mathcal{Y}(K)$ de formes quasi-modulaires de Hilbert pour $\Gamma_{K}$; il s'agit de fonctions holomorphes de $n$ variables complexes $z_{1}, \ldots, z_{n}$.

Notons :

$$
\mathcal{D}=\left\{D_{1}, \ldots, D_{n}\right\}=\left\{(2 \pi \mathrm{i})^{-1} \partial / \partial z_{1}, \ldots,(2 \pi \mathrm{i})^{-1} \partial / \partial z_{n}\right\} .
$$

Nous verrons que $(\mathcal{Y}(K), \mathcal{D})$ est un anneau différentiel.

Nous aurons besoin de quelques propriétés élémentaires de cet anneau, que nous décrirons au paragraphe 2, et qui seront pour la plupart démontrées dans l'appendice. En particulier, si $n>1$, alors $\mathcal{Y}(K)$ n'est pas de type fini, contrairement au cas $K=\mathbb{Q}$, décrit dans [8]. Voici maintenant nos résultats concernant les idéaux premiers $\mathcal{D}$-stables de $\mathcal{Y}(K)$.

Théorème 2 Si $n>1$, alors l'anneau $\mathcal{Y}(K)$ ne possède pas d'idéaux principaux $\mathcal{D}$-stables non nuls. 
Nous avons obtenu des résultats partiels supplémentaires sur la structure des idéaux premiers différentiellement stables de $\mathcal{Y}(K)$ dans le cas où $K=$ $\mathbb{Q}(\sqrt{5})$.

Théorème 3 Supposons que $K=\mathbb{Q}(\sqrt{5})$; il existe une forme modulaire de Hilbert non nulle $\kappa$ avec la propriété suivante. Soit $\mathfrak{P}$ un idéal premier non nul D-stable de $\mathcal{Y}(K)$, supposons que $\mathfrak{P}$ contienne une forme modulaire de Hilbert non nulle; alors $\kappa \in \mathfrak{P}$.

Le théorème 3 laisse penser que la propriété de Ramanujan est valide pour $\mathcal{Y}(\mathbb{Q}(\sqrt{5}))$.

Voici un autre argument en faveur de cette hypothèse. Dans [12], nous avons démontré que l'anneau différentiel $\mathcal{Y}(\mathbb{Q})$ satisfait la propriété de Ramanujan. Tout idéal premier non nul et différentiellement stable contient la forme modulaire :

$$
\kappa(\mathbb{Q})=\operatorname{det}\left(\begin{array}{ll}
4 e_{4} & 6 e_{6} \\
\frac{d e_{4}}{d z} & \frac{d e_{6}}{d z}
\end{array}\right),
$$

où $e_{4}, e_{6}$ désignent les séries d'Eisenstein de poids 4,6 normalisées usuelles pour $\mathbf{S L}_{2}(\mathbb{Z})$.

La forme $\kappa(\mathbb{Q})$ est non nulle et proportionnelle à l'unique forme parabolique normalisée non nulle de poids 12 pour $\mathbf{S L}_{2}(\mathbb{Z}$ ) (donc proportionnelle à $\left.\Delta\left(e^{2 \pi \mathrm{i} t}\right), \Im(t)>0\right)$.

La forme $\kappa(\mathbb{Q})$ apparaît donc comme image d'un crochet de Rankin de deux générateurs de l'anneau des formes modulaires pour $\mathbf{S L}_{2}(\mathbb{Z})$, et cette expression joue un rôle important dans la démonstration de cette propriété.

Dans le cas où $K=\mathbb{Q}(\sqrt{5})$, un phénomène analogue apparaît. L'élément $\kappa=\kappa(\mathbb{Q}(\sqrt{5}))$ du théorème 3 s'exprime comme un crochet trilinéaire (voir [11] pour les propriétés de base de ces crochets) des générateurs $\varphi_{2}, \chi_{5}, \chi_{6}$ de l'anneau des formes modulaires de Hilbert de poids parallèle (cf. définition dans ce texte, ou [11]) :

$$
\kappa(\mathbb{Q}(\sqrt{5}))=\operatorname{det}\left(\begin{array}{ccc}
2 \varphi_{2} & 5 \chi_{5} & 6 \chi_{6} \\
\frac{\partial \varphi_{2}}{\partial z} & \frac{\partial \chi_{5}}{\partial z} & \frac{\partial \chi_{6}}{\partial z} \\
\frac{\partial \varphi_{2}}{\partial z^{\prime}} & \frac{\partial \chi_{5}}{\partial z^{\prime}} & \frac{\partial \chi_{6}}{\partial z^{\prime}}
\end{array}\right) .
$$


Les crochets multilinéaires (dont la définition générale apparaît dans [11], paragraphe 6.1) mettent en lumière une analogie entre les formes modulaires $\kappa(\mathbb{Q})$ et $\kappa(\mathbb{Q}(\sqrt{5}))$; de plus, dans $[11]$, nous avons démontré que $\kappa(\mathbb{Q}(\sqrt{5}))$ est proportionnelle à la forme modulaire $\chi_{15}$ construite par Gundlach dans [6]. Cette analogie a en partie déjà été remarquée par Resnikoff dans [14], car il avait noté que du point de vue différentiel, la série d'Eisenstein de poids $(2,2)$ normalisée $\varphi_{2}$ pour $\Gamma_{\mathbb{Q}(\sqrt{5})}$ joue un rôle similaire à celui de la série $E_{2}$. Dans ce texte, ces analogies seront rendues encore plus impressionantes, mais leur raison demeure obscure.

Ainsi, les expressions de $\kappa(\mathbb{Q})$ et $\kappa(\mathbb{Q}(\sqrt{5}))$ en termes de crochets multilinéaires n'expliquent pas complètement pourquoi ces formes modulaires jouent un rôle aussi privilégié et similaire dans les propriétés différentielles de $\mathcal{Y}(K)$ pour $K=\mathbb{Q}$ ou $K=\mathbb{Q}(\sqrt{5})$.

En lisant cet article, le lecteur verra qu'en partie, le théorème 3 est dû à une coïncidence que nous ne savons pas expliquer à présent, et que nous ésperons d'élucider dans des autres travaux.

\subsection{Plan de l'article.}

Voici le plan de cet article : il y a deux parties de longueur inégale, dont les contenus sont assez indépendants. Dans le paragraphe 2, après avoir défini l'anneau $\mathcal{Y}(K)$ et fait une description de ses propriétés de base, nous démontrerons le théorème 2 ; dans la plupart des résultats de ce paragraphe, $K$ est un corps de nombres totalement réel de dégré $n>1$ sur $\mathbb{Q}$.

La démonstration du théorème 3 occupera quant'à elle, tout le reste de l'article. Cette démonstration sera raménée à la démonstration d'un resultat concernant la structure différentielle d'anneaux de formes modulaires de Hilbert, ce qui explique la condition technique du théorème 3. Dans ces parties, le choix $K=\mathbb{Q}(\sqrt{5})$ sera fixé; les formes quasi-modulaires n'apparaissent pratiquement pas dans cette partie.

\section{Formes quasi-modulaires.}

Ici, nous définissons les formes quasi-modulaires de Hilbert et nous en présentons quelques propriétés élémentaires.

Soit $\mathcal{H}$ le demi-plan supérieur complexe, soit $K$ un corps de nombres totalement réel de degré $n$ sur $\mathbb{Q}$, soit $\sigma_{1}, \ldots, \sigma_{n}$ ses plongements dans $\mathbb{R}$, 
soit $\mathcal{O}_{K}$ son anneau d'entiers. Si $a \in K$, alors nous écrivons aussi $a_{i}=\sigma_{i}(a)$. Le groupe modulaire de Hilbert

$$
\Gamma_{K}=\mathbf{S L}_{2}\left(\mathcal{O}_{K}\right)
$$

agit sur $\mathcal{H}^{n}$ par transformations homographiques de la manière usuelle : soit $z=\left(z_{1}, \ldots, z_{n}\right) \in \mathcal{H}^{n}$ et $\gamma=\left(\begin{array}{ll}a & b \\ c d\end{array}\right) \in \mathbf{S L}_{2}\left(\mathcal{O}_{K}\right)$. Alors :

$$
\gamma(z)=\left(\frac{a_{1} z_{1}+b_{1}}{c_{1} z_{1}+d_{1}}, \ldots, \frac{a_{n} z_{n}+b_{n}}{c_{n} z_{n}+d_{n}}\right) .
$$

Remarquer que si $n=1$, alors $K=\mathbb{Q}$ et $\Gamma_{K}=\mathrm{SL}_{2}(\mathbb{Z})$.

Definition. Soit $F: \mathcal{H}^{n} \rightarrow \mathbb{C}$ une fonction holomorphe. On dit que $F$ est une forme quasi-modulaire de Hilbert de poids $\left(k_{1}, \ldots, k_{n}\right) \in \mathbb{Z}^{n}$ et profondeur $s \in \mathbb{N}$ si

(i) $n=1$ et $F$ est une forme quasi-modulaire pour $\mathbf{S L}_{2}(\mathbb{Z})$ comme défini par Kaneko et Zagier dans [8], ou

(ii) $n>1$, et il existe un polynôme $P \in \operatorname{Hol}\left(\mathcal{H}^{n}\right)\left[X_{1}, \ldots, X_{n}\right]$ de degré total $s$ en les indéterminées $X_{1}, \ldots, X_{n}$ (dont les coefficients sont des fonctions holomorphes sur $\left.\mathcal{H}^{n}\right)$, tel que, pour tout $z \in \mathcal{H}^{n}$ et $\gamma \in \Gamma_{K}$ :

$$
F(\gamma(z)):=\prod_{i=1}^{n}\left(c_{i} z_{i}+d_{i}\right)^{k_{i}} P\left(\frac{c_{1}}{c_{1} z_{1}+d_{1}}, \ldots, \frac{c_{n}}{c_{n} z_{n}+d_{n}}\right) .
$$

On voit facilement, en utilisant le fait que $F$ admet une expansion en série de Fourier, que le terme constant de $P$ par rapport à $X_{1}, \ldots, X_{n}$ est égal à $F$.

La notion de forme quasi-modulaire de Hilbert généralise la notion de forme modulaire de Hilbert. Par exemple, une forme quasi-modulaire de Hilbert de profondeur 0 est une forme modulaire. De plus, si $F$ est une forme quasi-modulaire de poids $\left(k_{1}, \ldots, k_{n}\right)$ et profondeur $s$, alors $F(z, \ldots, z)$ est une forme quasi-modulaire de poids $\sum_{i} k_{i}$ et profondeur $n s$ pour $\mathbf{S L}_{2}(\mathbb{Z})$.

Si $F$ est une forme quasi-modulaire de Hilbert de poids $\underline{k}:=\left(k_{1}, \ldots, k_{n}\right)$, alors $D_{i} F$ est une forme quasi-modulaire de Hilbert de poids

$$
\underline{k}+2_{i}:=\left(k_{1}, \ldots, k_{i-1}, k_{i}+2, k_{i+1}, \ldots, k_{n}\right) .
$$

Donc $(\mathcal{Y}(\mathcal{K}), \mathcal{D})$ est un anneau différentiel. 
Des formes quasi-modulaires de Hilbert non constantes et de poids distincts sont $\mathbb{C}$-linéairement indépendants, donc les éspaces vectoriels complexes $\mathcal{Y}(K)_{\left(k_{1}, \ldots, k_{n}\right)}$ engendrés par les formes quasi-modulaires de Hilbert de poids $\left(k_{1}, \ldots, k_{n}\right)$ sont linéairement disjoints, filtrés par les profondeurs, et l'anneau $\mathcal{Y}(K)$ des polynômes en des formes quasi-modulaires de Hilbert de tout poids entier et toute profondeur est multi-gradué par les poids :

$$
\mathcal{Y}(K)=\bigoplus_{\left(k_{1}, \ldots, k_{n}\right) \in \mathbb{Z}^{n}} \mathcal{Y}(K)_{\left(k_{1}, \ldots, k_{n}\right)}
$$

Démonstration du théorème 2. Supposons par l'absurde qu'il existe deux éléments $A, F \in \mathcal{Y}(K)$ et une dérivation $D_{i} \in \mathcal{D}$ avec :

$$
D_{i} F=A F
$$

Nous pouvons supposer que $F$ soit une fonction non constante, ce qui implique $A \neq 0$. Il existe des formes quasi-modulaires de Hilbert $F_{\underline{k}}$ non nulles, de poids distincts $\underline{k}$ et $A_{\underline{h}}$ de poids distincts $\underline{h}$, de telle sorte que :

$$
F=\sum_{\underline{k}} F_{\underline{k}}, \quad A=\sum_{\underline{h}} A_{\underline{h}},
$$

les sommes étant finies. Dans l'ensemble $\left\{F_{\underline{k}}\right\}$, choisissons un élément non constant $F_{\underline{k}_{0}}$, tel que le poids $\underline{k}_{0}=\left(k_{0,1}, \ldots, k_{0, n}\right)$ satisfasse la propriété suivante : le nombre $v\left(\underline{k}_{0}\right):=\sum_{j \neq i} k_{0, j}$ est le plus petit possible, et $k_{0, i}$ est le plus grand possible pour ce choix de $v\left(\underline{k}_{0}\right)$.

On en déduit que l'ensemble $\left\{A_{\underline{h}}\right\}$ contient un élément non nul $A_{2_{i}}$, de poids

$$
2_{i}:=(\underbrace{0, \ldots, 0}_{i-1 \text { fois }}, 2,0, \ldots, 0),
$$

tel que :

$$
D_{i} F_{\underline{k}_{0}}=A_{2_{i}} F_{\underline{k}_{0}} .
$$

Ceci constitue une contradiction avec le lemme 11 de l'appendice.

Dans l'appendice nous démontrons également le résultat suivant, qui est aussi une conséquence simple du lemme 11.

Théorème 4 Si $[K: \mathbb{Q}]>1$, alors l'anneau $\mathcal{Y}(K)$ des formes quasi-modulaires de Hilbert pour $\Gamma_{K}$ n'est pas de type fini. 
Ce théorème pour $n=2$ est aussi un corollaire d'un résultat de N. Oled Aaiez concernant la structure différentielle des formes quasi-modulaires non holomorphes, associées à des cycles de Hirzebruch-Zagier compacts sur $\mathcal{H}^{2}$ (travail à paraître).

\section{Propriété de Ramanujan de formes modu- laires.}

Dans les paragraphes qui suivent, nous munissons un anneau de formes modulaires de Hilbert de deux variables complexes (associé au corps $K=$ $\mathbb{Q}(\sqrt{5}))$ de plusieurs dérivations liées aux crochets de Rankin. L'anneau différentiel ainsi obtenu satisfait lui aussi la propriété de Ramanujan; ceci impliquera le théorème 3 .

Faisons tout de suite un survol un peu plus précis de ce que nous allons montrer. Soit $\mathcal{O}_{K}$ l'anneau des entiers de $K$. Nous allons définir, sur l'anneau $\mathcal{T}$ engendré par les formes modulaires de Hilbert de poids parallèles pour le groupe $\Gamma=\Gamma_{K}$, un ensemble de dérivations

$$
\mathfrak{D}=\left\{d_{1}, d_{2}, e_{1}, e_{2}, f_{1}, f_{2}\right\} .
$$

Nous verrons que $(\mathcal{T}, \mathfrak{D})$ satisfait la propriété de Ramanujan, mais que cet anneau n'a pas d'idéaux premiers principaux non nuls et stables, contrairement à l'anneau $(\mathcal{R}, D)$ (il y a «trop de dérivations $\gg)$.

Nous verrons qu'en définissant

$$
\mathfrak{D}^{*}=\left\{\left(d_{1}+d_{2}\right) / 2,\left(e_{1}+e_{2}\right) / 2,\left(f_{1}+f_{2}\right) / 2\right\},
$$

l'anneau différentiel $\left(\mathcal{T}, \mathfrak{D}^{*}\right)$ satisfait lui aussi la propriété de Ramanujan, et a de plus un, et un seul idéal premier principal non nul $\mathfrak{D}^{*}$-stable (il y a le «bon nombre de dérivations »).

En revanche, l'anneau différentiel $\left(\mathcal{T},\left\{e_{1}+e_{2}\right\}\right)$, possède deux idéaux premiers non nuls stables $\left(\chi_{5}\right)$ et $\left(\chi_{15}\right)$, mais nous ne savons pas démontrer que cet anneau différentiel satisfait la propriété de Ramanujan (Il n'y a pas « assez de dérivations »).

Voici les résultats principaux de cette partie, que nous préferons enoncer avant même de décrire les objets utilisés.

Théorème 5 Si $\mathcal{Q}$ est un idéal premier non nul de $\mathcal{T}$ qui est $\mathfrak{D}$-stable, alors $\chi_{15} \in \mathcal{Q}$. 
Nous allons montrer qu'il existe un sous-anneau $\mathcal{T}^{*}$ de $\mathcal{T}$, isomorphe à l'anneau de polynômes $\mathbb{C}\left[X_{1}, X_{2}, X_{3}\right]$ (pour des indéterminées $X_{1}, X_{2}, X_{3}$ ), tel que $\left(\mathcal{T}^{*}, \mathfrak{D}^{*}\right)$ est un anneau différentiel. On verra que $\chi_{15} \notin \mathcal{T}^{*}$, mais que

$$
\chi=\chi_{15}^{2} \in \mathcal{T}^{*},
$$

et que ce polynôme est irréductible.

Théorème 6 Si $\mathcal{P}$ est un idéal premier non nul de $\mathcal{T}^{*}$ qui est $\mathfrak{D}^{*}$-stable, alors $\chi \in \mathcal{P}$.

Nous donnerons une description plus détaillée de ces anneaux différentiels ; pour l'instant, il est utile de savoir que le théorème 5 s'obtient directement comme corollaire du théorème 6 , et que le théorème 3 n'est qu'un corollaire du théorème 5 .

Des calculs numériques plus approfondis nous ont permis de préciser les théorèmes 5 et 6 ; ceci sera abordé dans la proposition 4 du paragraphe 5 . Les démonstrations que nous donnerons mettent en relief des analogies formelles entre l'anneau différentiel $(\mathcal{R}, D)$ et l'anneau différentiel $\left(\mathcal{T}^{*}, \mathfrak{D}^{*}\right)$.

\subsection{Structure et dérivations modulaires.}

Pour les notions élémentaires concernant les formes modulaires de Hilbert associées au corps $K=\mathbb{Q}(\sqrt{5})$, nous utiliserons essentiellement [11] : nous en reprenons en grande partie les notations et la terminologie. Nous designons souvent par $\underline{r}$ un couple de nombres complexes $\left(r_{1}, r_{2}\right)$. Si $n$ est un entier, nous posons $n_{1}=(n, 0)$ et $n_{2}=(0, n)$ : nous écrirons par exemple $2_{1}=(2,0)$.

Soit $M_{\underline{r}}(\Gamma)$ l'espace vectoriel des formes modulaires de Hilbert de poids $\underline{r}$. Nous avons :

$$
\mathcal{T}=\bigoplus_{n \in \mathbb{N}} M_{(n, n)}(\Gamma)
$$

Posons aussi :

$$
\mathcal{L}=\bigoplus_{\underline{r} \in \mathbb{N}^{2}} M_{\underline{r}}(\Gamma) .
$$

$\mathcal{L}$ est l'anneau des polynômes en des formes modulaires de Hilbert de tout poids (non nécessairement parallèle) : contrairement à $\mathcal{T}$, cet anneau n'est pas de type fini (cf. [11], lemme 16). 
Dans la suite, nous considérons le poids d'une forme modulaire de poids parallèle comme un entier, plutôt que comme un couple d'entiers.

Nous désignons par $\mathcal{T}_{\text {sym }}$ le sous-anneau de $\mathcal{T}$ dont les éléments sont les formes modulaires $F$ telles que $F \circ \mathcal{C}=F$, où $\mathcal{C}$ est la symétrie $\mathcal{C}\left(z, z^{\prime}\right)=$ $\left(z^{\prime}, z\right)$ : c'est le sous-anneau des formes modulaires symétriques. Une forme modulaire $F$ est dite antisymétrique si elle satisfait $F \circ \mathcal{C}=-F$.

Sur les espaces vectoriels de formes modulaires de Hilbert $M_{\underline{\underline{r}}}$ sont définis certains opérateurs différentiels, qui sont des avatars des crochets de Rankin et Rankin-Cohen. Soit $F$ une forme modulaire de Hilbert de poids $\underline{f}=$ $\left(f_{1}, f_{2}\right)$. Nous utiliserons les opérateurs non linéaires suivants :

$$
\begin{aligned}
(2 \pi i)^{2} \Pi_{i} F & :=f_{i} F \frac{\partial^{2} F}{\partial z_{i}^{2}}-\left(f_{i}+1\right)\left(\frac{\partial F}{\partial z_{i}}\right)^{2} \\
(2 \pi i)^{2} \Lambda F & :=F \frac{\partial^{2} F}{\partial z_{1} \partial z_{2}}-\frac{\partial F}{\partial z_{1}} \frac{\partial F}{\partial z_{2}}
\end{aligned}
$$

Un calcul direct sur les facteurs d'automorphie, et la détermination du terme constant dans les expansions en série de Fourier, montrent que :

$$
\begin{aligned}
\Pi_{i}: M_{\underline{f}}(\Gamma) & \rightarrow S_{2 \underline{f}+4_{i}}(\Gamma), \\
\Lambda: M_{\underline{f}}(\Gamma) & \rightarrow S_{2 \underline{f}+(2,2)}(\Gamma),
\end{aligned}
$$

où $S_{\underline{r}}(\Gamma)$ est l'espace vectoriel des formes paraboliques de poids $\underline{r}$.

Soit $G$ une forme modulaire de Hilbert de poids $\underline{g}=\left(g_{1}, g_{2}\right)$ : nous posons :

$$
(2 \pi i)[F, G]_{1_{i}}:=f_{i} F \frac{\partial G}{\partial z_{i}}-g_{i} G \frac{\partial F}{\partial z_{i}}=\operatorname{det}\left(\begin{array}{l}
f_{i} F g_{i} G \\
\frac{\partial F}{\partial z_{i}} \frac{\partial G}{\partial z_{i}}
\end{array}\right) .
$$

Nous avons un opérateur bilinéaire :

$$
[\cdot, \cdot]_{1_{i}}: M_{\underline{f}}(\Gamma) \times M_{\underline{g}}(\Gamma) \rightarrow S_{\underline{f}+\underline{g}+2_{i}}(\Gamma) .
$$

Soient $F, G, H$ trois formes modulaires de Hilbert de poids parallèles $f, g, h$ respectivement. L'opérateur :

$$
(2 \pi \mathrm{i})^{2}[F, G, H]=\frac{(g+h)}{2} \operatorname{det}\left(\begin{array}{ccc}
f F & g G & h H \\
\frac{\partial F}{\partial z} & \frac{\partial G}{\partial z} & \frac{\partial H}{\partial z} \\
\frac{\partial F}{\partial z^{\prime}} & \frac{\partial G}{\partial z^{\prime}} & \frac{\partial H}{\partial z^{\prime}}
\end{array}\right)
$$


est trilinéaire et nous avons :

$$
[\cdot, \cdot, \cdot]: M_{f}(\Gamma) \times M_{g}(\Gamma) \times M_{h}(\Gamma) \rightarrow S_{f+g+h+2}(\Gamma) .
$$

On a aussi l'identité :

$$
[F, G, H]=\frac{1}{2}\left(\left[F,[G, H]_{1_{2}}\right]_{1_{1}}-\left[F,[G, H]_{1_{1}}\right]_{1_{2}}\right),
$$

voir [11], proposition 3. Un fait très important à remarquer est que ces crochets ne sont pas bien définis sur des polynômes quelconque en des formes modulaires, mais seulement sur les formes modulaires, puisque ils dépendent du poids. On a le résultat suivant (cf. théorème 6.1 de [11], voir aussi [7]) :

Proposition 1 Soit $\varphi_{2}$ la série d'Eisenstein de poids 2 pour $\Gamma$, normalisée pour que le terme constant de son développement de Fourier à l'infini soit égal à 1 . Il existe trois formes paraboliques non nulles $\chi_{5}, \chi_{6}, \chi_{15}$ de poids parallèles respectivement $5,6,15$, avec $\chi_{6}, \chi_{15}$ (et $\varphi_{2}$ ) symétriques et $\chi_{5}$ antisymétrique, satisfaisant les propriétés suivantes.

$$
\begin{aligned}
\chi_{6} & =\frac{1}{24} \Lambda \varphi_{2}, \\
\chi_{5}^{2} & =\frac{-1}{2880} \varphi_{2}^{-1}\left(\left(\Pi_{1} \varphi_{2}\right)\left(\Pi_{2} \varphi_{2}\right)-9\left(\Lambda \varphi_{2}\right)\right), \\
\chi_{15} & =\frac{\sqrt{5}}{22}\left[\chi_{6}, \varphi_{2}, \chi_{5}\right] .
\end{aligned}
$$

L'anneau des formes modulaires de Hilbert pour le groupe $\Gamma$, est égal à l'anneau (gradué par les poids) des polynômes isobares en $\varphi_{2}, \chi_{5}, \chi_{6}, \chi_{15}$, divisé par l'idéal engendré par la rélation :

$$
\chi_{15}^{2}=\chi
$$

avec

$$
\begin{aligned}
\chi= & \lambda\left(50000 \chi_{5}^{6}-1000 \varphi_{2}^{2} \chi_{6} \chi_{5}^{4}+\varphi_{2}^{5} \chi_{5}^{4}-2 \varphi_{2}^{4} \chi_{6}^{2} \chi_{5}^{2}+\right. \\
& \left.+1800 \varphi_{2} \chi_{6}^{3} \chi_{5}^{2}+\varphi_{2}^{3} \chi_{6}^{4}-864 \chi_{6}^{5}\right)
\end{aligned}
$$

et $\lambda=484 / 49$. 
Sur $\mathcal{T}$ on a des involutions $\iota, \varsigma$, définies par :

$$
\varsigma\left(\chi_{5}\right)=-\chi_{5}, \quad \iota\left(\chi_{15}\right)=-\chi_{15} .
$$

Nous écrirons souvent $\bar{x}:=\iota(x)$ : l'involution $\iota$ est $\mathcal{T}^{*}$-linéaire (où $\mathcal{T}^{*}=$ $\left.\mathbb{C}\left[\varphi_{2}, \chi_{5}, \chi_{6}\right]\right)$ et associe à une forme modulaire de Hilbert $F\left(z, z^{\prime}\right)$ de poids parallèle, la forme modulaire $F\left(\epsilon^{2} z^{\prime}, \epsilon^{\prime 2} z\right)$, où

$$
\epsilon=\frac{1+\sqrt{5}}{2}
$$

L'involution $\varsigma$ est $\mathcal{T}_{\text {sym }}$-linéaire, et associe à une forme modulaire de Hilbert $F\left(z, z^{\prime}\right)$ de poids parallèle, la forme modulaire $F\left(z^{\prime}, z\right)$, composée de $F$ avec la symétrie $\mathcal{C}$ (noter que $\mathcal{T}_{\text {sym }}=\mathbb{C}\left[\varphi_{2}, \chi_{5}^{2}, \chi_{6}, \chi_{15}\right]$, d'après la proposition 1 ).

Remarque. nous avons normalisé $\varphi_{2}, \chi_{5}, \chi_{6}, \chi_{15}$ de telle sorte que tous les coefficients de Fourier de leurs expansions à la pointe à l'infini de la surface modulaire de Hilbert associée à $K$ soient des entiers rationnels premiers entre eux. Ce choix n'est pas celui de [7] ou [5]. C'est pourquoi la relation $\chi_{15}^{2}-\chi$ avec $\chi$ comme dans (3), ne ressemble pas à la formule (16) p. 109 de [7] ou à la formule du corollaire 2.2 p. 191 de [5].

\subsection{Les six dérivations.}

Nous présentons ici les 6 dérivations $d_{1}, d_{2}, e_{1}, e_{2}, f_{1}, f_{2}$. Soient $G, H$ deux formes modulaires dans $\mathcal{T}$ de poids $g, h$. Les applications :

$$
\begin{aligned}
M_{f}(\Gamma) & \rightarrow S_{f+g+h+2} \\
F & \mapsto\left[F,[G, H]_{1_{1}}\right]_{1_{2}} \\
F & \mapsto\left[F,[G, H]_{1_{2}}\right]_{1_{1}}
\end{aligned}
$$

sont définies sur les éléments de $\mathcal{T}$ qui sont homogènes pour la graduation de la proposition 1 (des éléments isobares, c'est-à-dire des formes modulaires de poids parallèle).

Considérons le cas où $G, H \in\left\{\varphi_{2}, \chi_{5}, \chi_{6}\right\}$. Pour tout $r$, nous avons six applications linéaires sur $M_{r}(\Gamma)$ ainsi définies :

$$
\begin{aligned}
d_{1}(X)=\left[X,\left[\varphi_{2}, \chi_{5}\right]_{1_{2}}\right]_{1_{1}} & d_{2}(X)=\left[X,\left[\varphi_{2}, \chi_{5}\right]_{1_{1}}\right]_{1_{2}} \\
e_{1}(X)=\left[X,\left[\varphi_{2}, \chi_{6}\right]_{1_{2}}\right]_{1_{1}} & e_{2}(X)=\left[X,\left[\varphi_{2}, \chi_{6}\right]_{1_{1}}\right]_{1_{2}} \\
f_{1}(X)=\left[X,\left[\chi_{5}, \chi_{6}\right]_{1_{2}}\right]_{1_{1}} & f_{2}(X)=\left[X,\left[\chi_{5}, \chi_{6}\right]_{1_{1}}\right]_{1_{2}} .
\end{aligned}
$$


Ces applications sont isobares de poids parallèles 9, 10,13, ou en d'autres termes :

$$
\begin{aligned}
& d_{1}, d_{2}: M_{r}(\Gamma) \rightarrow S_{r+9}(\Gamma) \\
& e_{1}, e_{2}: M_{r}(\Gamma) \rightarrow S_{r+10}(\Gamma), \\
& f_{1}, f_{2}: M_{r}(\Gamma) \rightarrow S_{r+13}(\Gamma)
\end{aligned}
$$

Posons

$$
d^{*}=\left(d_{1}+d_{2}\right) / 2, \quad e^{*}=\left(e_{1}+e_{2}\right) / 2, \quad f^{*}=\left(f_{1}+f_{2}\right) / 2,
$$

et

$$
d_{*}=\left(d_{1}-d_{2}\right) / 2, \quad e_{*}=\left(e_{1}-e_{2}\right) / 2, \quad f_{*}=\left(f_{1}-f_{2}\right) / 2,
$$

de telle sorte que $d_{1}=d^{*}+d_{*}, d_{2}=d^{*}-d_{*}$, etc. Posons aussi

$$
\mathfrak{D}^{*}=\left\{d^{*}, e^{*}, f^{*}\right\}, \quad \mathfrak{D}_{*}=\left\{d_{*}, e_{*}, f_{*}\right\} .
$$

Si $F, G \in \mathcal{T}$ sont deux formes modulaires de poids $f, g$, alors $d_{1}(F G)=$ $F d_{1}(G)+G d_{1}(F)$. Ainsi l'application linéaire $d_{1}$ se prolonge par linéarité en une dérivation de l'anneau $\mathcal{T}$; si $F=\sum_{l=0}^{k} F_{l}$ est un élément de $\mathcal{T}$ avec $F_{l}$ forme modulaire de Hilbert de poids parallèle $l$, alors nous posons :

$$
d_{1}(F)=\sum_{l=1}^{k} d_{1}\left(F_{l}\right) .
$$

Par le même argument, tous les éléments de $\mathfrak{D}, \mathfrak{D}^{*}, \mathfrak{D}_{*}$ se prolongent en des dérivations sur l'anneau $\mathcal{T}$, compatibles avec la graduation par les poids des formes modulaires.

Soit $\mathcal{T}_{0}=\mathbb{C}\left[\varphi_{2}, \chi_{5}^{2}, \chi_{6}\right]$. La proposition 1 dit que $\mathcal{T}$ a une structure de $\mathcal{T}_{0}$-module libre de rang 4 . Plus précisement on a

$$
\mathcal{T}=\mathcal{T}_{0} \oplus \chi_{5} \mathcal{T}_{0} \oplus \chi_{15} \mathcal{I}_{0} \oplus \chi_{5} \chi_{15} \mathcal{I}_{0}
$$

En particulier, $\mathcal{I}_{\text {sym }}=\mathcal{T}_{0} \oplus \chi_{15} \mathcal{I}_{0}$ et $\mathcal{T}^{*}=\mathcal{T}_{0} \oplus \chi_{5} \mathcal{I}_{0}$. Dans la proposition qui suit, nous nous servons de cette $\mathcal{T}_{0}$-structure pour déterminer les images des sous-modules $\mathcal{T}_{\text {sym }}$ et $\mathcal{T}^{*}$ et des autres, par les opérateurs de dérivation de $\mathfrak{D}^{*}$ ou de $\mathfrak{D}_{*}$. 
Proposition 2 On a les inclusions suivantes.

$$
\begin{aligned}
d^{*}\left(\mathcal{T}_{\text {sym }}\right), e^{*}\left(\chi_{5} \mathcal{T}_{\text {sym }}\right), f^{*}\left(\mathcal{T}_{\text {sym }}\right) & \subset \chi_{5} \mathcal{T}_{\text {sym }} \\
d^{*}\left(\chi_{5} \mathcal{T}_{\text {sym }}\right), e^{*}\left(\mathcal{T}_{\text {sym }}\right), f^{*}\left(\chi_{5} \mathcal{T}_{\text {sym }}\right) & \subset \mathcal{T}_{\text {sym }} \\
d_{*}\left(\mathcal{T}_{\text {sym }}\right), e_{*}\left(\chi_{5} \mathcal{T}_{\text {sym }}\right), f_{*}\left(\mathcal{T}_{\text {sym }}\right) & \subset \mathcal{T}_{\text {sym }} \\
d_{*}\left(\chi_{5} \mathcal{T}_{\text {sym }}\right), e_{*}\left(\mathcal{T}_{\text {sym }}\right), f_{*}\left(\chi_{5} \mathcal{T}_{\text {sym }}\right) & \subset \chi_{5} \mathcal{T}_{\text {sym }} \\
d^{*}\left(\mathcal{T}^{*}\right), e^{*}\left(\mathcal{T}^{*}\right), f^{*}\left(\mathcal{T}^{*}\right) & \subset \mathcal{T}^{*} \\
d^{*}\left(\chi_{15} \mathcal{T}^{*}\right), e^{*}\left(\chi_{15} \mathcal{T}^{*}\right), f^{*}\left(\chi_{15} \mathcal{T}^{*}\right) & \subset \chi_{15} \mathcal{T}^{*} \\
d_{*}\left(\mathcal{T}^{*}\right), e_{*}\left(\mathcal{T}^{*}\right), f_{*}\left(\mathcal{T}^{*}\right) & \subset \chi_{15} \mathcal{T}^{*} \\
d_{*}\left(\chi_{15} \mathcal{T}^{*}\right), e_{*}\left(\chi_{15} \mathcal{T}^{*}\right), f_{*}\left(\chi_{15} \mathcal{T}^{*}\right) & \subset \mathcal{T}^{*} .
\end{aligned}
$$

De plus, on a les égalités:

$$
d_{2}(x)=\overline{d_{1}(\bar{x})}, e_{2}(x)=\overline{e_{1}(\bar{x})}, f_{2}(x)=\overline{f_{1}(\bar{x})} .
$$

Démonstration. Notons que pour une fonction $X$ suffisamment dérivable :

$$
\frac{\partial}{\partial z_{1}}(X \circ \mathcal{C})=\left(\frac{\partial X}{\partial z_{2}}\right) \circ \mathcal{C}, \quad \frac{\partial}{\partial z_{2}}(X \circ \mathcal{C})=\left(\frac{\partial X}{\partial z_{1}}\right) \circ \mathcal{C}
$$

Soit $\rho \in \mathcal{T}$ une forme modulaire non nulle de poids $r$, symétrique ou antisymétrique, posons $\delta(\rho)=1$ si $\rho$ est symétrique et $\delta(\rho)=-1$ si $\rho$ est antisymétrique. Alors :

$$
\frac{\partial \rho}{\partial z_{1}}=\delta(r)\left(\frac{\partial \rho}{\partial z_{2}}\right) \circ \mathcal{C}
$$

On en déduit que $\left[\varphi_{2}, \chi_{5}\right]_{1_{1}} \circ \mathcal{C}=-\left[\varphi_{2}, \chi_{5}\right]_{1_{2}},\left[\varphi_{2}, \chi_{6}\right]_{1_{1}} \circ \mathcal{C}=\left[\varphi_{2}, \chi_{6}\right]_{1_{2}}$, et $\left[\chi_{5}, \chi_{6}\right]_{1_{1}} \circ \mathcal{C}=-\left[\chi_{5}, \chi_{6}\right]_{1_{2}}$. 
Calculons maintenant $d_{2}(\rho) \circ \mathcal{C}$ :

$$
\begin{aligned}
& d_{2}(\rho) \circ \mathcal{C}= \\
&=\quad\left[\rho,\left[\varphi_{2}, \chi_{5}\right]_{1_{1}}\right]_{1_{2}} \circ \mathcal{C} \\
&=\quad r(\rho \circ \mathcal{C})\left(\left(\frac{\partial}{\partial z_{2}}\left[\varphi_{2}, \chi_{5}\right]_{1_{1}}\right) \circ \mathcal{C}\right)- \\
& 7\left(\left[\varphi_{2}, \chi_{5}\right]_{1_{1}} \circ \mathcal{C}\right)\left(\left(\frac{\partial \rho}{\partial z_{2}}\right) \circ \mathcal{C}\right) \\
&= \delta(\rho)\left(r \rho \frac{\partial}{\partial z_{1}}\left(\left[\varphi_{2}, \chi_{5}\right]_{1_{1}} \circ \mathcal{C}\right)-7\left(-\left[\varphi_{2}, \chi_{5}\right]_{1_{2}}\right) \frac{\partial \rho}{\partial z_{1}}\right) \\
&= \delta(\rho)\left(-r \rho \frac{\partial}{\partial z_{1}}\left[\varphi_{2}, \chi_{5}\right]_{1_{2}}+7\left[\varphi_{2}, \chi_{5}\right]_{1_{2}} \frac{\partial \rho}{\partial z_{1}}\right) \\
&=-\delta(\rho)\left[\rho,\left[\varphi_{2}, \chi_{5}\right]_{1_{2}}\right]_{1_{1}}=-\delta(\rho) d_{1}(\rho) .
\end{aligned}
$$

Une formule analogue est valide pour $d_{2}(\rho) \circ \mathcal{C}$. Donc si $s$ est une forme modulaire symétrique, alors $d^{*}(s)$ est antisymétrique : elle appartient donc à $\chi_{5} \mathcal{T}_{\text {sym }}$. De même, $d_{*}(s)$ est symétrique. Si $a$ est une forme modulaire antisymétrique, alors $d^{*}(a)$ est symétrique. En raisonnant de la même façon pour les autres dérivations $e^{*}, e_{*}, f^{*}, f_{*}$, on démontre toutes les inclusions concernant $\mathcal{T}_{\text {sym }}$ et $\chi_{5} \mathcal{T}_{\text {sym }}$.

Les inclusions concernant $\mathcal{T}^{*}$ et $\chi_{15} \mathcal{T}^{*}$ en découlent aussi, car d'après la proposition 1, toute forme modulaire de Hilbert symétrique de poids parallèles impairs est produit d'une forme modulaire symétrique de poids pairs et de $\chi_{15}$. Comme $d^{*}, f^{*}$ envoient des formes modulaires de poids pair sur des formes modulaires de poids impair, et $e^{*}$ envoie des formes modulaires de poids pair sur des formes modulaires de poids impair, on obtient toutes les images des sous-modules liés à $\mathcal{T}_{\text {sym }}$ et à $\mathcal{T}^{*}$.

Ceci implique que pour tout $r \in \mathcal{T}$ on a

$$
\overline{d^{*}(\bar{r})}=d^{*}(r), \quad \overline{d_{*}(\bar{r})}=-d_{*}(r) .
$$

On en déduit les égalités (5). La démonstration de la proposition 2 est terminée.

Corollaire 1 L'idéal principal de $\mathcal{T}$ engendré par $\chi_{15}$ est $\mathfrak{D}^{*}$-stable. L'idéal engendré par $\chi_{5}$ est $e^{*}$-stable.

Démonstration. On utilise la collection d'inclusions (4); on en déduit les inclusions $d^{*}\left(\chi_{15}\right), e^{*}\left(\chi_{15}\right), f^{*}\left(\chi_{15}\right) \in \chi_{15} \mathcal{T}^{*}$. 
Si $e^{*}\left(\chi_{5}\right)$ est non nulle, alors elle a poids 15 , et est antisymetrique d'après la proposition 2. Comme toutes les formes modulaires de $\mathcal{T}$ de poids $\leq 14$ sont dans $\mathcal{T}^{*}$ (cf. proposition 1 ), on trouve $e^{*}\left(\chi_{5}\right) \in \chi_{5} \mathcal{I}_{0}$. Le lemme qui suit implique que $e^{*}\left(\chi_{5}\right) \neq 0$.

Lemme 1 Si $t \in \mathfrak{D}, y \in \mathcal{T}$ et $t y=0$, alors $y \in \mathbb{C}$. Si $t^{*} \in \mathfrak{D}, x \in \mathcal{T}$ et $t^{*} x=0$, alors $x \in \mathbb{C}$.

Démonstration. Pour démontrer ces propriétés, il suffit de le faire avec $x, y$ des formes modulaires, car toutes les dérivations sont isobares.

Démontrons la première propriété avec $t=d_{1}$ : la démonstration donnée s'étend facilement aux autres dérivations de $\mathfrak{D}$.

Soit $y$ une forme modulaire telle que $d_{1} y=0$. On a $\left[y,\left[\varphi_{2}, \chi_{5}\right]_{1_{2}}\right]_{1_{1}}=0$. Ceci implique que $y$ et $\left[\varphi_{2}, \chi_{5}\right]_{1_{2}}$ sont multiplicativement dépendants modulo $\mathbb{C}^{\times}$d'après le lemme 11 de $[11]$, mais ceci n'est possible que si $y \in \mathbb{C}$, car $y$ est de poids parallèles, alors que $\left[\varphi_{2}, \chi_{5}\right]_{1_{2}}$ ne l'est pas.

Soit maintenant $x \in \mathcal{T}$ une forme modulaire telle que $d^{*} x=0$. On peut écrire $x=s+a$, où $s$ est une forme modulaire symétrique, et $a$ est une forme modulaire antisymétrique. On a $d^{*}(s+a)=d^{*} s+d^{*} a=0$. D'après la proposition $2, d^{*} s$ est antisymétrique et $d^{*} a$ est symétrique. Donc, on a $d^{*} s=0$ et $d^{*} a=0$.

Il suffit de démontrer que pour toute forme modulaire symétrique $s$, si $d^{*} s=0$ alors $s \in \mathbb{C}$, et que pour toute forme modulaire antisymétrique $a$, si $d^{*} a=0$ alors $a=0$.

Soit $s$ une forme modulaire symétrique non constante, telle que $d^{*} s=0$. Si $d_{*} s=0$, alors $d_{1} s=d_{2} s=0$, d'où $s \in \mathbb{C}$, d'après la première partie du lemme. Donc $d_{*} s \neq 0$. On a $d_{*} s=d_{1} s=-d_{2} s$, car $d_{1}=d_{*}+d^{*}$ et $d_{2}=d_{*}-d^{*}$. Donc, d'après (5) :

$$
\begin{aligned}
d_{*} s & =d_{1} s=-d_{2} s \\
& =-\iota d_{1} \iota s \\
& =-\iota d_{1} s \\
& =-\iota d_{*} s \\
& =-d_{*} s,
\end{aligned}
$$

car $d_{*}$ preserve la symétrie (proposition 2), d'où une contradiction. Donc $d^{*} s \neq 0$. 
Soit $a$ une forme modulaire antisymétrique non constante, telle que $d^{*} a=$ 0 . Si $d_{*} a=0$, alors $d_{1} a=d_{2} a=0$, d'où $a \in \mathbb{C}$ et donc $a=0$ car elle est antisymétrique. Donc $d_{*} a \neq 0$. On a $d_{*} a=d_{1} a=-d_{2} a$ et donc, d'après (5):

$$
\begin{aligned}
d_{*} a & =d_{1} a=-d_{2} a \\
& =-\iota d_{1} \iota a \\
& =\iota d_{1} a \\
& =\iota d_{*} a \\
& =-d_{*} a,
\end{aligned}
$$

d'où une contradiction. Donc $d^{*} a \neq 0$. La même démonstration fonctionne avec la dérivation $f^{*}$, et à des petites modifications près, avec $e^{*}$.

\section{Idéaux différentiellement stables.}

Dans ce paragraphe nous démontrons les théorèmes 5 et 6 : commençons par le théorème 6 .

La démonstration du théorème 6 procède en plusieurs étapes. Nous déterminons (1) les idéaux principaux stables pour $\mathfrak{D}^{*},(2)$ les idéaux premiers de $\mathcal{T}^{*}$ qui sont $\mathfrak{D}^{*}$-stables, n'ayant pas d'éléments dans $\mathbb{C}\left[\varphi_{2}\right] \cup \mathbb{C}\left[\chi_{5}\right] \cup \mathbb{C}\left[\chi_{6}\right]$, (3) les idéaux premiers de $\mathcal{T}^{*}$ qui sont $\mathfrak{D}^{*}$-stables, et qui contiennent au moins un élément non nul de $\mathbb{C}\left[\varphi_{2}\right] \cup \mathbb{C}\left[\chi_{5}\right] \cup \mathbb{C}\left[\chi_{6}\right]$.

\subsection{Idéaux principaux $\mathfrak{D}^{*}$-stables.}

Lemme 2 L'idéal $(\chi)$ est l'unique idéal premier principal non nul de $\mathcal{T}^{*}$, qui est $\mathfrak{D}^{*}$-stable.

Démonstration. Le corollaire 1 implique que $(\chi)$ est $\mathfrak{D}^{*}$-stable : en effet, pour tout $t^{*} \in \mathfrak{D}^{*}, t^{*}(\chi)=t^{*}\left(\chi_{15}^{2}\right)=2 \chi_{15} t^{*}\left(\chi_{15}\right)$. Or, $t^{*}\left(\chi_{15}\right) \in \chi_{15} \mathcal{T}^{*}$ (corollaire 1 ), donc $t^{*}(\chi) \in \chi \mathcal{T}^{*}$. C'est facile de démontrer que $(\chi)$ est un idéal premier de $\mathcal{T}^{*}$.

Nous démontrons que $(\chi)$ est le seul idéal premier principal $d^{*}$-stable de $\mathcal{T}^{*}$. Soit $p \in \mathcal{T}^{*}$ non constant tel que $\left(d^{*} p\right) \subset(p)$. En particulier, il existe $a \in \mathcal{T}^{*}$ tel que $d^{*} p=a p$. On peut alors écrire $: p=p_{m}+\cdots+p_{l}$ et $a=a_{h}+\cdots+a_{k}$ avec $p_{i}, a_{i}$ des formes modulaires de poids parallèles $i$, 
$m \leq l, h \leq k, p_{m}, p_{l} \neq 0$. Nous pouvons aussi supposer que $a_{h}, a_{k} \neq 0$ d'après le lemme 1 . On a :

$$
d^{*} p_{m}+\cdots+d^{*} p_{l}=a_{h} p_{m}+\cdots+a_{k} p_{l}
$$

Donc $d^{*} p_{m}=a_{h} p_{m}$ et $d^{*} p_{l}=a_{k} p_{l}$. Comme $d^{*}$ est de poids 9 , on trouve que $h=k=9$, et $a=a_{k}=a_{h}$. Donc $d^{*} p=a p$ et $a$ est une forme modulaire de poids parallèles 9 . Mais $M_{9}(\Gamma) \cong \mathbb{C} \varphi_{2}^{2} \chi_{5}$ (proposition 1 ), donc $a$ est un multiple non nul de $\varphi_{2}^{2} \chi_{5}$.

Ainsi, les deux formes modulaires $d^{*} \chi / \chi$ et $d^{*} p / p$ sont $\mathbb{C}$-linéairement dépendantes. On a donc :

$$
\frac{d^{*} \chi}{\chi}=\lambda \frac{d^{*} p}{p}
$$

avec $\lambda \in \mathbb{C}^{\times}$. Nous montrons maintenant que $\lambda \in \mathbb{Q}^{\times}$.

Jusqu'à présent, nous n'avons utilisé que des arguments algébriques : nous avons essentiellement travaillé avec des anneaux de polynômes. Il s'agit maintenant d'utiliser la structure analytique des formes modulaires.

Pour achever notre démonstration, il suffit de démontrer que si $F, G, H$ sont des formes modulaires de Hilbert algébriquement indépendantes, de poids parallèles respectivement $f, 2,5$, avec $H$ parabolique et $G(\infty)=1$, alors

$$
[F, G, H]^{*}=\lambda F G^{2} H
$$

implique $\lambda \in \mathbb{Q}$, où nous avons posé :

$$
[F, G, H]^{*}=\frac{1}{2}\left(\left[F,[G, H]_{1_{2}}\right]_{1_{1}}+\left[F,[G, H]_{1_{1}}\right]_{1_{2}}\right),
$$

car $d^{*} F=\left[F, \varphi_{2}, \chi_{5}\right]^{*}$ par définition. Nous utilisons les séries de Fourier du lemme 15 de [11] : ici $\mathcal{O}_{K,+}^{*}$ désigne l'ensemble des éléments totalement positifs du dual $\mathcal{O}_{K}^{*}$ de $\mathcal{O}_{K}$ pour la trace $\mathbf{t}$ de $K$ sur $\mathbb{Q}$. En particulier, en écrivant :

$$
\begin{aligned}
F\left(z, z^{\prime}\right) & =\sum_{\nu \in \mathcal{O}_{K,+}^{*} \cup\{0\}} f_{\nu} e\left(\nu z+\nu^{\prime} z^{\prime}\right), \\
G\left(z, z^{\prime}\right) & =\sum_{\nu \in \mathcal{O}_{K,+}^{*} \cup\{0\}} g_{\nu} e\left(\nu z+\nu^{\prime} z^{\prime}\right), \\
H\left(z, z^{\prime}\right) & =\sum_{\nu \in \mathcal{O}_{K,+}^{*}} h_{\nu} e\left(\nu z+\nu^{\prime} z^{\prime}\right),
\end{aligned}
$$


où $e(t)=e^{2 \pi \mathrm{it}},\left(z, z^{\prime}\right) \in \mathcal{H}^{2}$, et avec $f_{\nu}, g_{\nu}, h_{\nu} \in \mathbb{C}, g_{0}=1$, on trouve que

$$
\begin{aligned}
{[F, G, H]^{*} } & =\frac{1}{2}\left(\left[F,[G, H]_{1_{2}}\right]_{1_{1}}+\left[F,[G, H]_{1_{1}}\right]_{1_{2}}\right) \\
& =\sum_{\tau \in \mathcal{O}_{K,+}^{*}} e\left(\tau z+\tau^{\prime} z^{\prime}\right) \sum_{\alpha+\nu+\mu=\tau}^{\#} c_{\alpha, \nu, \mu} f_{\alpha} g_{\nu} h_{\mu},
\end{aligned}
$$

où la somme $\sum^{\sharp}$ est indexée par des éléments $(\alpha, \nu, \mu)$ de $\left(\mathcal{O}_{K,+}^{*} \cup\{0\}\right)^{3}$, et où

$$
c_{\alpha, \nu, \mu}:=\mathbf{t}\left(\left(f \alpha^{\prime}-7\left(\nu^{\prime}+\mu^{\prime}\right)\right)(2 \nu-5 \mu)\right) .
$$

De la même façon, on calcule :

$$
F G^{2} H=\sum_{\tau \in \mathcal{O}_{K,+}^{*}} e\left(\tau z+\tau^{\prime} z^{\prime}\right) \sum_{x+y+z+t=\tau}^{\sharp} f_{x} g_{y} g_{z} h_{t} .
$$

Supposons pour commencer que $F$ soit parabolique. Comme $F, H$ ne sont pas nulles, il existe deux entiers rationnels $k_{0}, h_{0}>0$ minimaux avec la propriété que pour quelques $\alpha \in \mathcal{O}_{K,+}^{*}$ avec $\mathbf{t}(\alpha)=k_{0}$, on ait $f_{\alpha} \neq 0$, et pour quelques $\mu \in \mathcal{O}_{K,+}^{*}$ avec $\mathbf{t}(\mu)=h_{0}$, on ait $h_{\mu} \neq 0$.

Soient maintenant $\alpha_{0}, \mu_{0} \in \mathcal{O}_{K,+}^{*}$ tels que $\mathbf{t}\left(\alpha_{0}\right)=k_{0}, \mathbf{t}\left(\mu_{0}\right)=h_{0}$, avec la propriété que $\alpha_{0}, \mu_{0}$ soient les plus grands possibles avec $f_{\alpha_{0}}, h_{\mu_{0}} \neq 0$ (On considère $K$ comme étant plongé dans $\mathbb{R}$ ), soit $\tau_{0}=\alpha_{0}+\mu_{0}$. L'expression (8) et le choix de $\tau_{0}$ impliquent que le coefficient de Fourier de $F G^{2} H$ associé à $\tau_{0}$ est égal à $g_{0}^{2} f_{\alpha_{0}} h_{\mu_{0}}=f_{\alpha_{0}} h_{\mu_{0}} \neq 0$.

De même, l'expression (7) et le choix de $\tau_{0}$ impliquent que le coefficient de Fourier de $[F, G, H]^{*}$ associé à $\tau_{0}$ est égal à

$$
\begin{aligned}
c_{\alpha_{0}, 0, \mu_{0}} g_{0} f_{\alpha_{0}} h_{\mu_{0}} & =c_{\alpha_{0}, 0, \mu_{0}} f_{\alpha_{0}} h_{\mu_{0}} \\
& =\mathbf{t}\left(5 \mu_{0}\left(f \alpha_{0}^{\prime}-7 \mu_{0}\right)\right) f_{\alpha_{0}} h_{\mu_{0}} .
\end{aligned}
$$

Mais $c_{\alpha, \nu, \mu}$ étant la trace d'un élément de $K$, c'est un nombre rationnel. Si $[F, G, H]^{*}=\lambda F G^{2} H$, alors $c_{\alpha_{0}, 0, \mu_{0}} f_{\alpha_{0}} h_{\alpha_{0}}=\lambda f_{\alpha_{0}} h_{\alpha_{0}}$, d'où $c_{\alpha_{0}, 0, \mu_{0}}=\lambda \in \mathbb{Q}$.

Supposons ensuite que $F$ ne soit pas parabolique : on a alors $f_{0} \neq 0$. Soit $\mu_{0}$ comme ci-dessus. Le coefficient de Fourier associé à $\mu_{0}$ dans (8) est $f_{0} h_{\mu_{0}}$, tandis que le coefficient de Fourier associé à $\mu_{0}$ dans (7) est $f_{0} h_{\mu_{0}} c_{0,0, \mu_{0}}=$ $-70 f_{0} h_{\mu_{0}} \mathbf{n}\left(\mu_{0}\right)$ : on en déduit que $\lambda \in \mathbb{Q}^{\times}$. 
Nous avons donc démontré l'existence de deux entiers rationnels $u, v$ tels que $d^{*}\left(p^{u} \chi^{v}\right) /\left(p^{u} \chi^{v}\right)=0$ (où $d^{*}$ designe l'extension de la dérivation au corps des fractions de $\left.\mathcal{T}^{*}\right)$, ou de manière équivalente, $d^{*}\left(p^{u} \chi^{v}\right)=0$. Le lemme 1 implique $p^{u} \chi^{v} \in \mathbb{C}$. On trouve donc $(p)=(\chi)$, et le lemme 2 est démontré.

Remarques. Dans la démonstration du lemme 2 nous avons utilisé le fait remarquable que $\operatorname{dim}_{\mathbb{C}} M_{9}(\Gamma)=1$. L'unicité d'un idéal principal $t^{*}$-stable pour une dérivation $t^{*}$ est fausse en général. Par exemple, l'opérateur $e^{*}$ possède l'idéal principal premier stable $\left(\chi_{5}\right)$.

Observons aussi que $\chi_{5}, \chi_{15}$ sont des générateurs du $\mathcal{T}_{0}$-module libre $\mathcal{T}$. Dans [3] et [4], on fait des hypothèses très générales quant'à la structure des anneaux des formes modulaires. L'hypothèse p. 88 de [4] affirme que, pour tout anneau $\mathfrak{I}$ de formes modulaires (dans une classe considérée par Baily et Borel dans [1]), il existe un certain sous-module $\mathfrak{H}$ tel que $\mathfrak{I}$ soit un $\mathfrak{H}$-module libre. Ce serait très intéressant de comparer cette structure avec la structure différentielle de $\mathfrak{I}$.

\subsection{Idéaux premiers $\mathfrak{D}^{*}$-stables de hauteur 2 .}

Soient $F, G, H$ trois formes modulaires de Hilbert algébriquement indépendantes, de poids parallèles $f, g, h$. D'après la proposition 3 de [11], la forme modulaire $[F, G, H]$, de poids parallèles $f+g+h+2$ est non nulle.

La formule suivante est élémentaire et peut se démontrer avec un calcul direct (nous l'avons trouvée en suivant les techniques introduites dans [16]; un logiciel de calcul symbolique quelconque permet de la vérifier) :

$$
\begin{aligned}
&(f+g)(f+h)[F, G, H]^{2}= \\
&=(f+g)(g+h)\left([F, F, G]^{*}[H, G, H]^{*}-[F, G, H]^{*}[H, F, G]^{*}\right)- \\
&(g+h)(f+h)\left([H, G, H]^{*}[G, F, G]^{*}-[H, F, G]^{*}[G, F, H]^{*}\right)+ \\
&(g+h)^{2}\left([F, F, H]^{*}[G, G, H]^{*}-[F, G, H]^{*}[G, F, H]^{*}\right) .
\end{aligned}
$$

Soit $M$ la matrice :

$$
M=\left(\begin{array}{ccc}
{[G, G, H]^{*}} & {[H, G, H]^{*}} & {[F, G, H]^{*}} \\
{[G, G, F]^{*}} & {[H, G, F]^{*}} & {[F, G, F]^{*}} \\
{[G, H, F]^{*}} & {[H, H, F]^{*}} & {[F, H, F]^{*}}
\end{array}\right) .
$$

La formule (9) implique qu'elle a rang $\geq 2$. En effet, l'indépendance algébrique de $F, G, H$ implique, en utilisant la proposition 3 de [11], que $[F, G, H] \neq 0$, 
donc $[F, G, H]^{2} \neq 0$. Or, l'expression à droite de (9) est une combinaison linéaire de mineurs de taille 2 de la matrice $M$. Ainsi, un mineur de $M$ de taille 2 est non nul $\left(^{4}\right)$.

Supposons que $F=\chi_{6}, G=\varphi_{2}$ et $H=\chi_{5}$. Alors

$$
M=\left(\begin{array}{ccc}
d^{*} \varphi_{2} & d^{*} \chi_{5} & d^{*} \chi_{6} \\
e^{*} \varphi_{2} & e^{*} \chi_{5} & e^{*} \chi_{6} \\
f^{*} \varphi_{2} & f^{*} \chi_{5} & f^{*} \chi_{6}
\end{array}\right) .
$$

Soit $\tilde{M}$ la matrice des mineurs de $M$ de taille 2. D'après le raisonnement précedant, cette matrice possède des coefficients non nuls. La matrice suivante décrit les poids des coefficients de $M$ :

$$
\left(\begin{array}{lll}
11 & 14 & 15 \\
12 & 15 & 16 \\
15 & 18 & 19
\end{array}\right)
$$

Donc les coefficients de $\tilde{M}$ sont tous des formes modulaires de Hilbert, et les poids sont repartis de la manière suivante :

$$
\left(\begin{array}{lll}
34 & 31 & 30 \\
33 & 30 & 29 \\
30 & 27 & 26
\end{array}\right)
$$

(on notera que tous les coefficients de l'adjointe $\hat{M}$ de $M$ ont même poids 45). Nous avons le lemme suivant.

Lemme 3 Soit $\mathcal{P}$ un idéal premier non principal de $\mathcal{T}^{*}$ tel que $\mathcal{P} \cap\left(\mathbb{C}\left[\varphi_{2}\right] \cup\right.$ $\left.\mathbb{C}\left[\chi_{5}\right] \cup \mathbb{C}\left[\chi_{6}\right]\right)=(0)$. Si $\mathcal{P}$ est $\mathfrak{D}^{*}$-stable, alors tous les coefficients de $\tilde{M}$ appartiennent à $\mathcal{P}$. De plus, $\chi \in \mathcal{P}$.

Démonstration. Les hypothèses impliquent qu'il existe dans $\mathcal{P}$, trois polynômes irréductibles $A, B, C$ avec les propriétés suivantes :

$$
\begin{array}{ll}
\frac{\partial A}{\partial \chi_{6}}=0, & \frac{\partial A}{\partial \varphi_{2}} \frac{\partial A}{\partial \chi_{5}} \neq 0 \\
\frac{\partial B}{\partial \chi_{5}}=0, & \frac{\partial B}{\partial \varphi_{2}} \frac{\partial B}{\partial \chi_{6}} \neq 0 \\
\frac{\partial C}{\partial \varphi_{2}}=0, & \frac{\partial C}{\partial \chi_{5}} \frac{\partial C}{\partial \chi_{6}} \neq 0 .
\end{array}
$$

\footnotetext{
${ }^{4}$ On peut démontrer que si $F, G, H$ sont des formes modulaires de poids parallèle algébriquement indépendantes, alors $M$ est de rang 3 , mais nous ne le ferons pas ici.
} 
On peut même supposer que les degrés partiels de ces polynômes soient les plus petits possibles, car il n'y a pas d'idéal non trivial de $\mathcal{T}^{*}$ qui soit stable pour les trois dérivées partielles $\partial / \partial \varphi_{2}, \partial / \partial \chi_{5}, \partial / \partial \chi_{6}$.

On a $d^{*} A, d^{*} B, \ldots, f^{*} C \in \mathcal{P}$. En particulier, $d^{*} A, e^{*} A \in \mathcal{P}$, et

$$
\left(d^{*} A\right)\left(e^{*} \varphi_{2}\right)-\left(e^{*} A\right)\left(d^{*} \varphi_{2}\right) \in \mathcal{P} \text {. }
$$

On vérifie l'égalité :

$$
\left(d^{*} A\right)\left(e^{*} \varphi_{2}\right)-\left(e^{*} A\right)\left(d^{*} \varphi_{2}\right)=\frac{\partial A}{\partial \chi_{5}}\left(d^{*} \chi_{5} e^{*} \varphi_{2}-e^{*} \chi_{5} d^{*} \varphi_{2}\right) .
$$

Comme $\partial A / \partial \chi_{5} \notin \mathcal{P}$, on a $d^{*} \chi_{5} e^{*} \varphi_{2}-e^{*} \chi_{5} d^{*} \varphi_{2} \in \mathcal{P}$. De la même façon que ci-dessus, on vérifie alors que tous les coefficients de la matrice $\tilde{M}=\left(\tilde{m}_{i, j}\right)_{i, j}$ appartiennent à $\mathcal{P}$.

La dernière propriété du lemme se démontre en utilisant la formule (9), car $\left[\chi_{6}, \varphi_{2}, \chi_{5}\right]^{2}$ est proportionnel à $\chi$ (avec constante de proportionnalité non nulle), et égal à une combinaison linéaire non triviale des cofficients qui se trouvent sur l'anti-diagonale de $\tilde{M}$.

Remarque. Les arguments de ce paragraphe pourraient se généraliser au cas où $K$ est un autre corps quadratique réel différent de $\mathbb{Q}(\sqrt{5})$.

\subsection{Détermination explicite de rélations différentielles.}

Pour continuer, nous devons expliciter les rélations engendrées par les dérivations de $\mathfrak{D}^{*}$. Nous utilisons la proposition suivante.

Proposition 3 On a les rélations suivantes.

$$
\begin{gathered}
d^{*}\left(\varphi_{2}\right)=\frac{4}{5} \chi_{5}\left(\varphi_{2}^{3}-1050 \chi_{6}\right) \\
d^{*}\left(\chi_{6}\right)=-\frac{2}{5} \chi_{5}\left(\varphi_{2}^{2} \chi_{6}+875 \chi_{5}^{2}\right) \\
d^{*}\left(\chi_{5}\right)=\frac{1}{10} \varphi_{2}\left(7 \chi_{6}^{2}-15 \varphi_{2} \chi_{5}^{2}\right) \\
e^{*}\left(\varphi_{2}\right)=-1152 \chi_{6}^{2}+240 \varphi_{2} \chi_{5}^{2}+\frac{4}{5} \varphi_{2}^{3} \chi_{6} \\
e^{*}\left(\chi_{6}\right)=-240 \chi_{5}^{2} \chi_{6}-\frac{8}{5} \varphi_{2}^{2} \chi_{6}^{2}+\frac{4}{5} \varphi_{2}^{3} \chi_{5}^{2} \\
e^{*}\left(\chi_{5}\right)=\chi_{5}\left(-\frac{6}{5} \varphi_{2}^{2} \chi_{6}+200 \chi_{5}^{2}\right)
\end{gathered}
$$




$$
\begin{aligned}
f^{*}\left(\varphi_{2}\right) & =\chi_{5}\left(550 \chi_{5}^{2}-\frac{4}{5} \varphi_{2}^{2} \chi_{6}\right) \\
f^{*}\left(\chi_{6}\right) & =\chi_{5}\left(\frac{11}{4} \varphi_{2}^{2} \chi_{5}^{2}-\frac{59}{20} \varphi_{2} \chi_{6}^{2}\right) \\
f^{*}\left(\chi_{5}\right) & =\chi_{6}\left(\frac{7}{2} \varphi_{2} \chi_{5}^{2}-\frac{33}{10} \chi_{6}^{2}\right) .
\end{aligned}
$$

Esquisse de démonstration. Montrons comment obtenir la première des rélations de la proposition 3 .

Nous savons, d'après le lemme 1 , que $d^{*}\left(\varphi_{2}\right)$ est non nulle. Il s'agit d'une forme modulaire de poids parallèle 11 . La proposition 1 implique que :

$$
d^{*}\left(\varphi_{2}\right)=\alpha \chi_{5} \varphi_{2}^{3}+\beta \chi_{5} \chi_{6}, \quad \alpha, \beta \in \mathbb{C} .
$$

Cette relation linéaire implique l'existence de relations linéaires entre coefficients de Fourier de $d^{*}\left(\varphi_{2}\right), \chi_{5} \varphi_{2}^{3}, \chi_{5} \chi_{6}$, et le calcul explicite de quelques coefficient de Fourier est suffisant pour calculer $\alpha, \beta$. Pour les autres relations aussi, on effectue une résolution numérique de systèmes linéaires, en utilisant la proposition 1.

Plue en général, il faut calculer un certain nombre de coefficients de Fourier de $\varphi_{2}, \chi_{5}, \chi_{6}, \chi_{15}$. Pour ce faire il suffit, d'après les relations différentielles de la proposition 1, de connaître tous les coefficients de Fourier à l'infini de $\varphi_{2}, \chi_{5}$, correspondants à des éléments totalement positifs du dual pour la trace de l'anneau des entiers de $K$, de trace majorée par un entier assez grand (nous les appellons coefficients de « petite trace »).

La connaissance de ces coefficients de Fourier de $\varphi_{2}$ nous permet de déterminer une partie de la série de Fourier de $\chi_{6}$ et de $\chi_{5}^{2}$. Pour calculer les coefficients de Fourier de petite trace de $\varphi_{2}$, on peut appliquer les formules de [11].

Pour calculer les coefficients de Fourier de petite trace de $\chi_{5}$, on peut appliquer la formule explicite de [2]. Tous ces coefficients permettent à leur tour de calculer, via le crochet $[\cdot, \cdot, \cdot]$, les coefficients de Fourier de petite trace pour $\chi_{15}$.

La méthode est la même que dans [11] ou [14], nous ne donnons pas les détails de calcul ici : le logiciel «Pari »permet de vérifier facilement toutes les rélations de la proposition, et nous rémercions D. Simon pour avoir vérifié toutes ces formules. 
Remarque. Nous pouvons expliciter les rélations du corollaire 1 grâce à la proposition 3 :

$$
\begin{aligned}
d^{*} \chi & =-2 \varphi_{2}^{2} \chi_{5} \chi \\
e^{*} \chi & =-4\left(\varphi_{2}^{2} \chi_{6}-300 \chi_{5}^{2}\right) \chi \\
f^{*} \chi & =-\varphi_{2} \chi_{5} \chi_{6} \chi .
\end{aligned}
$$

Dans toute la suite, nous allons utiliser les rélations décrites explicitement par la proposition 3.

\subsection{Idéaux premiers $\mathfrak{D}^{*}$-stables de hauteur 3 .}

Dans ce paragraphe, nous étudions les idéaux de $\mathcal{T}^{*}$ qui ont intersection non nulle avec l'ensemble $\mathbb{C}\left[\varphi_{2}\right] \cup \mathbb{C}\left[\chi_{5}\right] \cup \mathbb{C}\left[\chi_{6}\right]$; nous verrons qu'ils ont tous hauteur $\geq 3$.

Lemme 4 Soit $\mathcal{P}$ un idéal premier de $\mathcal{T}^{*}$, supposons qu'au moins une des conditions suivantes soit vérifiée :

$$
\mathcal{P} \cap \mathbb{C}\left[\varphi_{2}\right] \neq(0), \quad \mathcal{P} \cap \mathbb{C}\left[\chi_{5}\right] \neq(0), \quad \mathcal{P} \cap \mathbb{C}\left[\chi_{6}\right] \neq(0) .
$$

Si $\mathcal{P}$ est $\mathfrak{D}^{*}$-stable, alors $\mathcal{P}$ contient l'idéal premier $\left(\chi_{5}, \chi_{6}\right)$. En particulier, $\chi \in \mathcal{P}$.

Démonstration. Comme $\mathcal{P}$ est premier, on a ou bien $\varphi_{2}-c_{2} \in \mathcal{P}$, ou bien $\chi_{5}-c_{5} \in \mathcal{P}$, ou bien $\chi_{6}-c_{6} \in \mathcal{P}$, pour $c_{2}, c_{5}, c_{6} \in \mathbb{C}$.

(1). Supposons d'abord que $\chi_{5}-c_{5} \in \mathcal{P}$ : nous montrons que $\chi_{6} \in \mathcal{P}$. On a :

$$
d^{*} \chi_{5}, f^{*} \chi_{5}, d^{* 2} \chi_{5}, f^{* 2} \chi_{5} \in \mathcal{P}
$$

car pour tout $t^{*} \in \mathfrak{D}^{*}, t^{*}(\mathbb{C})=0$. En appliquant la proposition 3 , on peut calculer explicitement les polynômes (11).

Pour vérifier les calculs suivants, il est conseillé de se servir d'un logiciel de calcul symbolique. On calcule les résultants :

$$
\begin{aligned}
\operatorname{Rés}_{\chi_{5}}\left(d^{*} \chi_{5}, d^{* 2} \chi_{5}\right) & =\xi_{1} \varphi_{2}^{5} \chi_{6}^{6} A_{1} \in \mathcal{P} \\
\operatorname{Rés}_{\chi_{5}}\left(f^{*} \chi_{5}, f^{* 2} \chi_{5}\right) & =\xi_{2} \chi_{6}^{15} A_{2} \in \mathcal{P},
\end{aligned}
$$


où $\xi_{1}, \xi_{2} \in \mathbb{Q}^{\times}$, et où $A_{1}, A_{2} \in \mathbb{C}\left[\varphi_{2}, \chi_{6}\right]$ désignent des polynômes isobares non nuls de poids 12 . On vérifie que $A_{1}, A_{2}$ sont premiers entre eux, et pour des raisons de poids, ils sont égaux à des produits de la forme

$$
\prod_{s}\left(a_{s} \varphi_{2}^{3}-\chi_{6}\right)
$$

où l'on vérifie que $a_{s} \in \overline{\mathbb{Q}}^{\times}$pour tout $s$.

Comme $\mathcal{P}$ est premier, dans chacun des deux polynômes de (12) et (13) il existe au moins un facteur irréductible appartenant à $\mathcal{P}$; si $\chi_{6} \in \mathcal{P}$ nous avons terminé, supposons donc par l'absurde que $\chi_{6} \notin \mathcal{P}$.

Ainsi, $\mathcal{P}$ contient au moins un facteur de $A_{2}$, d'après (13), et ne peut pas contenir $\varphi_{2}$ d'après (12), car sinon, en éliminant $\varphi_{2}$ d'un facteur de la forme (14), nous obtenons $\chi_{6} \in \mathcal{P}$. Donc $\mathcal{P}$ contient $A_{1}, A_{2}$, et comme il est premier, il contient aussi deux facteurs irréductibles premiers entre eux de la forme (14). En éliminant $\varphi_{2}$, nous parvenons une fois de plus à une contradiction, car nous obtenons $\chi_{6} \in \mathcal{P}$.

(2). Supposons que $\chi_{6}-c_{6} \in \mathcal{P}$ : nous montrons que $\chi_{5} \in \mathcal{P}$. On a que :

$$
d^{*} \chi_{6}, d^{* 2} \chi_{6}, e^{*} f^{*} \chi_{6} \in \mathcal{P} .
$$

On calcule cette fois les résultants suivants, qui appartiennent tous à $\mathcal{P}$ :

$$
\begin{aligned}
\operatorname{Rés}_{\chi_{6}}\left(d^{*} \chi_{6}, d^{* 2} \chi_{6}\right) & =\xi_{3} \varphi_{2}^{3} \chi_{5}^{7} B_{1} \in \mathcal{P} \\
\operatorname{Rés}_{\chi_{6}}\left(d^{*} \chi_{6}, e^{*} f^{*} \chi_{6}\right) & =\xi_{4} \chi_{5}^{9} B_{2} \in \mathcal{P},
\end{aligned}
$$

où $\xi_{3}, \xi_{4} \in \mathbb{Q}^{\times}$et $B_{1}, B_{2}$ sont deux polynômes isobares non nuls de $\mathbb{C}\left[\varphi_{2}, \chi_{5}\right]$ de poids 10,20 .

On vérifie que $B_{1}, B_{2}$ sont premiers entre eux; pour de raisons de poids, ce sont des produits de la forme :

$$
\prod_{s}\left(b_{s} \varphi_{2}^{5}-\chi_{5}^{2}\right)
$$

avec $b_{s} \in \overline{\mathbb{Q}}^{\times}$pour tout $s$. En suivant la technique appliquée au point (1), on obtient $\chi_{5} \in \mathcal{P}$.

Dans les deux cas (1) ou (2) on voit que l'idéal $\left(\chi_{5}, \chi_{6}\right)$ est contenu dans $\mathcal{P}$. En effet, d'après $(\mathbf{1})$, si $\chi_{5} \in \mathcal{P}$, alors $\chi_{6} \in \mathcal{P}$ et $\left(\chi_{5}, \chi_{6}\right) \subset \mathcal{P}$. Si parcontre 
$\chi_{5}-c_{5} \in \mathcal{P}$ et $c_{5} \neq 0$, alors on trouve $\chi_{6} \in \mathcal{P}$ d'après (1), puis $\chi_{5} \in \mathcal{P}$ d'après (2), et finalement, $\mathcal{P}=\mathcal{T}^{*} \supset\left(\chi_{5}, \chi_{6}\right)$.

(3). Supposons pour terminer que $\varphi_{2}-c_{2} \in \mathcal{P}$. On calcule les résultants :

$$
\begin{aligned}
& \operatorname{Rés}_{\varphi_{2}}\left(d^{*} \varphi_{2}, d^{* 2} \varphi_{2}\right)=\xi_{5} \chi_{5}^{11} C_{1} \in \mathcal{P} \\
& \operatorname{Rés}_{\varphi_{2}}\left(e^{*} \varphi_{2}, e^{* 2} \varphi_{2}\right)=\xi_{6} \chi_{5}^{8} C_{2} \in \mathcal{P},
\end{aligned}
$$

avec $\xi_{5}, \xi_{6} \in \mathbb{Q}^{\times}$, et $C_{1}, C_{2}$ deux polynômes isobares non nuls de poids 30,60 , premiers entre eux de la forme

$$
\prod_{s}\left(d_{s} \chi_{5}^{6}-\chi_{6}^{5}\right)
$$

avec $d_{s} \in \overline{\mathbb{Q}}^{\times}$pour tout $s$. En utilisant la même technique des deux points précedants, on voit que $\chi_{5} \in \mathcal{P}$, d'où $\left(\chi_{5}, \chi_{6}\right) \subset \mathcal{P}$ grâce au point (1).

Dans tous les cas, $\left(\chi_{5}, \chi_{6}\right) \subset \mathcal{P}$. Comme $\chi \in\left(\chi_{5}, \chi_{6}\right)$ (formule $\left.(3)\right)$, le lemme 4 est entièrement démontré.

\subsection{Preuve des théorèmes 3,5 et 6 .}

Démonstration du théorème 6. Soit $\mathcal{P}$ un idéal premier non trivial de $\mathcal{T}^{*}, \mathfrak{D}^{*}$-stable. Sa hauteur géométrique $h$ varie dans l'ensemble $\{1,2,3\}$. Si $h=1$ alors $\mathcal{P}$ est principal, donc égal à $(\chi)$ d'après le lemme 2. Supposons maintenant que $h \geq 2$. Si $\mathcal{P} \cap\left(\mathbb{C}\left[\varphi_{2}\right] \cup \mathbb{C}\left[\chi_{5}\right] \cup \mathbb{C}\left[\chi_{6}\right]\right) \neq(0)$, d'après le lemme 4 on a $\chi \in \mathcal{P}$, et la proposition est démontrée dans ce cas.

Nous pouvons donc supposer que $\mathcal{P} \cap\left(\mathbb{C}\left[\varphi_{2}\right] \cup \mathbb{C}\left[\chi_{5}\right] \cup \mathbb{C}\left[\chi_{6}\right]\right)=(0)$ et que $\mathcal{P}$ est non principal, et d'après le lemme $3, \chi \in \mathcal{P}$ : la preuve du théorème 6 est terminée.

Démonstration du théorème 5 . Nous devons d'abord énoncer trois lemmes techniques.

Lemme 5 Soit $\mathcal{Q}$ un idéal non nul de $\mathcal{T}$. Si $\mathcal{Q} \cap \overline{\mathcal{Q}}=(0)$, alors $\mathcal{Q}$ est principal.

Démonstration. Soit $\mathcal{Q}$ un idéal non principal de $\mathcal{T}$. Il existe deux éléments non nuls $p_{1}, p_{2}$ de $\mathcal{Q}$ tels que pour tout choix d'éléments $q_{1}, q_{2} \in \mathcal{T}$ non tous nuls, alors $q_{1} p_{1}+q_{2} p_{2} \neq 0$. Supposons par l'absurde que $\mathcal{Q} \cap \overline{\mathcal{Q}}=(0)$ : on a en particulier $\mathcal{Q} \cap \mathcal{T}^{*}=(0)$, car sinon, si $s \in \mathcal{Q} \cap \mathcal{T}$ est un élément non nul, alors $\bar{s}=s$ et $s \in \overline{\mathcal{Q}}$. 
Donc $p_{1}, p_{2} \notin \mathcal{T}^{*}$ : nous pouvons écrire alors $p_{1}=x_{1}+\chi_{15} y_{1}, p_{2}=x_{2}+$ $\chi_{15} y_{2}$, avec $x_{1}, x_{2}, y_{1}, y_{2} \in \mathcal{T}^{*}$, et $y_{1}, y_{2}$ non nuls. Mais $y_{2} p_{1}-y_{1} p_{2}=y_{2} x_{1}-$ $y_{1} x_{2} \neq 0$ est un élément de $\mathcal{Q}$ qui est dans $\mathcal{T}^{*}$, d'où une contradiction.

Lemme 6 Soit $\mathcal{Q}$ un idéal premier non nul de $\mathcal{T}$, ne contenant pas $\chi_{15}$, soit $\mathcal{P}=\mathcal{Q} \cap \mathcal{T}^{*}$. On $a$ :

$$
\mathcal{Q} \cap \overline{\mathcal{Q}}=\mathcal{P} \mathcal{T}
$$

Démonstration. C'est clair que $\mathcal{Q} \cap \overline{\mathcal{Q}} \supset \mathcal{P} \mathcal{T}$; montrons que $\mathcal{Q} \cap \overline{\mathcal{Q}} \subset \mathcal{P} \mathcal{T}$. Clairement, $\mathcal{Q} \cap \mathcal{T}^{*}=\overline{\mathcal{Q}} \cap \mathcal{T}^{*}$, car $\overline{\mathcal{T}^{*}}=\mathcal{T}^{*}$. D'autre part, $\mathcal{P}$ est un idéal premier de $\mathcal{T}^{*}$.

Soit $x \in \mathcal{Q} \cap \overline{\mathcal{Q}}$. On a $\bar{x} \in \mathcal{Q}$ et donc

$$
\beta:=x+\bar{x} \in \mathcal{P} \text {. }
$$

D'autre part, aussi $x-\bar{x} \in \mathcal{Q}:$ or $x-\bar{x} \in \chi_{15} \mathcal{T}^{*}$ et il existe $\alpha \in \mathcal{T}^{*}$ tel que $x-\bar{x}=\chi_{15} \alpha$; cet élément appartient à $\mathcal{Q}$.

Nous avons $\chi_{15}^{2}, \alpha \in \mathcal{T}^{*}$, donc $\chi_{15}^{2} \alpha \in \mathcal{P}$ Or, par hypothèse, $\chi_{15}^{2} \notin \mathcal{Q}$, donc $\chi_{15}^{2} \notin \mathcal{P}$ car $\mathcal{Q}$ est premier. Finalement, $\alpha \in \mathcal{P}$, d'où :

$$
\chi_{15} \alpha=x-\bar{x} \in \mathcal{P} \mathcal{T} .
$$

On a donc que $\beta$ et $\chi_{15} \alpha$ appartiennent à $\mathcal{P} \mathcal{T}$. Ainsi,

$$
x=\left(\beta+\chi_{15} \alpha\right) / 2 \in \mathcal{P} \mathcal{T},
$$

d'où $\mathcal{P} \mathcal{T} \supset \mathcal{Q} \cap \overline{\mathcal{Q}}$, d'où l'égalité (16) : le lemme 6 est démontré.

Lemme 7 Il existe des nombres $l_{1}, l_{2}, l_{3} \in K^{\times}$tels que si $r \in \mathcal{T}^{*}$, alors :

$$
d_{*} r=l_{1} \chi_{15} \frac{\partial r}{\partial \chi_{6}}, \quad e_{*} r=l_{2} \chi_{15} \frac{\partial r}{\partial \chi_{5}}, \quad f_{*} r=l_{3} \chi_{15} \frac{\partial r}{\partial \chi_{2}} .
$$

En particulier on $a$ :

$$
d_{*} \chi_{15}=\frac{l_{1}}{2} \frac{\partial \chi}{\partial \chi_{6}}, \quad e_{*} \chi_{15}=\frac{l_{2}}{2} \frac{\partial \chi}{\partial \chi_{5}}, \quad f_{*} \chi_{15}=\frac{l_{3}}{2} \frac{\partial \chi}{\partial \chi_{2}} .
$$


Démonstration. Utilisons la proposition 3 de [11]. On a $d_{*}\left(\varphi_{2}\right)=d_{*}\left(\chi_{5}\right)=$ 0 , et $d_{*}\left(\chi_{6}\right)=(7 / 2)\left[\chi_{6}, \varphi_{2}, \chi_{5}\right] \neq 0$, car $\chi_{6}, \varphi_{2}, \chi_{5}$ sont algébriquement indépendantes. Donc $d_{*}\left(\chi_{6}\right)$ est proportionnel à $\chi_{15}$ pour une constante de proportionnalité non nulle : plus précisement, on a $d_{*}\left(\chi_{6}\right)=(11 / \sqrt{5}) \chi_{15}$. Les autres formules de (17) s'obtiennent de la même façon. Les constantes $l_{1}, l_{2}, l_{3}$ peuvent se calculer explicitement.

Les formules explicites de (18), s'obtiennent tout simplement en calculant $d_{*}(\chi), e_{*}(\chi), f_{*}(\chi)$, car on a, par exemple pour la dérivation $d_{*}: d_{*}\left(\chi_{15}^{2}\right)=$ $2 \chi_{15} d_{*}\left(\chi_{15}\right)$.

Preuve du théorème 5 . Soit $\mathcal{Q}$ un idéal premier de $\mathcal{T}$, stable pour tous les opérateurs de $\mathfrak{D}$. On a que $\overline{\mathcal{Q}}$ est $\mathfrak{D}$-stable, d'après (5). En particulier, les idéaux $\mathcal{Q}$ et $\overline{\mathcal{Q}}$ sont $\mathfrak{D}^{*}$-stables. Comme $\mathfrak{D}^{*} \mathcal{T}^{*} \subset \mathcal{T}^{*}$, l'idéal $\mathcal{P}=\mathcal{Q} \cap \mathcal{T}^{*}$ est $\mathfrak{D}^{*}$-stable. Nous distinguons maintenant deux cas, suivant que $\mathcal{Q} \cap \overline{\mathcal{Q}}=(0)$ ou non.

(1). Supposons que $\mathcal{Q} \cap \overline{\mathcal{Q}}=(0)$. Alors, d'après le lemme 5 , $\mathcal{Q}$ est un idéal principal : soit $p$ un générateur : alors $p \notin \mathcal{T}^{*}$. Il existe six formes modulaires $a_{1}, a_{2}, b_{1}, b_{2}, c_{1}, c_{2}$, de poids parallèles respectivement $9,9,10,10,13,13$, telles que :

$$
d_{i} p=a_{i} p, \quad e_{i} p=b_{i} p, \quad f_{i} p=c_{i} p \text { pour } i=1,2 .
$$

En particulier, $a_{i}, b_{i}, c_{i} \in \mathcal{T}^{*}(i=1,2)$ et satisfont $\bar{a}_{i}=a_{i}, \ldots$, car toutes les formes modulaires de $\mathcal{T}$ de poids $\leq 14$ se trouvent dans $\mathcal{T}^{*}$, d'après la proposition 1 . D'après les égalités (5) :

$$
\begin{aligned}
& \overline{d_{1} p}=d_{2}(\bar{p})=a_{1} \bar{p} \\
& \overline{d_{2} p}=d_{1}(\bar{p})=a_{2} \bar{p},
\end{aligned}
$$

Posons $\theta=p \cdot \bar{p} \in \mathcal{T}^{*}$. On a :

$$
\begin{aligned}
d_{1} \theta & =\bar{p} d_{1}(p)+p d_{1}(\bar{p}) \\
& =a_{1} \theta+a_{2} \theta \\
& =\left(a_{1}+a_{2}\right) \theta \quad \text { et de manière analogue : } \\
d_{2} \theta & =\left(a_{2}+a_{1}\right) \theta .
\end{aligned}
$$

Donc, $d^{*} \theta=a \theta$, avec $a=a_{2}+a_{1}$, et on obtient, de la même façon : $e^{*} \theta=$ $b \theta, f^{*} \theta=c \theta$, avec $b=b_{1}+b_{2}$ et $c=c_{1}+c_{2}$. Donc, l'idéal $(\theta)$ de $\mathcal{T}^{*}$ est $\mathfrak{D}^{*}$-stable, égal à $\left(\chi^{l}\right)$ pour un certain entier $l$, d'après le lemme 2 . Soit $p^{\prime}$ 
un élément de $\mathcal{T}$ tel que $p^{\prime} \overline{p^{\prime}}=\chi$. Alors $p^{\prime}$ est une forme modulaire, et proportionnel à $\chi_{15}$. Donc $l=1$ et $\theta \in \mathbb{C}^{\times} \chi$.

Nous avons démontré que si $\mathcal{Q}$ est un idéal principal $\mathfrak{D}$-stable, alors $\mathcal{Q}=$ $\left(\chi_{15}\right)$. En utilisant l'égalité $(18)$ du lemme 7 , on vérifie que que $\left(\chi_{15}\right)$ n'est pas $\mathfrak{D}_{*}$-stable : comme il est quand-même $\mathfrak{D}^{*}$-stable, on en déduit que $\left(\chi_{15}\right)$ n'est pas $\mathfrak{D}$-stable. Nous avons démontré en fait :

Lemme 8 Il n'existe pas d'idéal principal non nul $\mathfrak{D}$-stable dans $\mathcal{T}$.

(2). Supposons que $\mathcal{Q} \cap \overline{\mathcal{Q}} \neq(0)$. D'après le lemme $6, \mathcal{P}$ est non nul, et premier; il est de plus $\mathfrak{D}^{*}$-stable. Supposons par l'absurde que $\chi_{15} \notin \mathcal{Q}$.

Comme $\mathcal{P}$ est $\mathfrak{D}^{*}$-stable, il contient $\chi$ d'après le théorème 6. Donc $\chi \in \mathcal{Q}$ et $\chi_{15} \in \mathcal{Q}$ : une contradiction. La démonstration du théorème 5 est terminée.

Preuve du théorème 3 . Soit $\mathfrak{P}$ un idéal premier stable de $\mathcal{Y}(K)$, contenant une forme modulaire de Hilbert non nulle. Alors l'idéal $\mathcal{Q}$ engendré par toutes les formes modulaires de $\mathfrak{P}$ est un idéal premier non nul et $\mathfrak{D}$-stable de $\mathcal{T}$, qui contient $\chi_{15}$ d'après le théorème 5 .

\section{$5 \quad$ Un résultat plus précis.}

Soient $a, b$ deux nombres complexes, considérons l'idéal

$$
\mathcal{P}(a, b)=\left(a \varphi_{2}^{5}-\chi_{5}^{2}, b \varphi_{2}^{3}-\chi_{6}\right)
$$

de $\mathcal{T}^{*}$ et l'idéal

$$
\mathcal{Q}(a, b)=\left(\mathcal{P}(a, b), \chi_{15}\right)
$$

de $\mathcal{T}$. Ici nous donnons une esquisse de démonstration du résultat suivant.

Proposition 4 Si $\mathcal{P}$ est un idéal premier non nul de $\mathcal{T}^{*}$ qui est $\mathfrak{D}^{*}$-stable et de hauteur $\geq 2$, alors $\mathcal{P}$ contient $\mathcal{P}(a, b)$ avec :

$$
(a, b) \in \mathcal{E}:=\left\{\left(\frac{1}{800000}, \frac{1}{800}\right),\left(\frac{1}{253125}, \frac{1}{675}\right),(0,0)\right\} .
$$

Si $\mathcal{Q}$ est un idéal premier non nul de $\mathcal{T}$ qui est $\mathfrak{D}$-stable, alors il contient $\mathcal{Q}(a, b)$ avec $(a, b) \in \mathcal{E}$. 
La proposition 4 est certes plus précise des théorèmes 5 et 6 , mais la démonstration que nous en donnons utilise de manière essentielle la proposition 3 , ce qui ne peut que limiter des éventuelles généralisations.

Nous avons besoin du lemme qui suit, dont la démonstration est seulement esquissée, et dépend fortement des formules explicites de la proposition 3.

Lemme 9 Nous avons les propriétés suivantes.

1. Si $a, b \in \mathbb{C}^{\times}$, ou si $a=b=0$, l'idéal $\mathcal{P}(a, b)$ est primaire de hauteur 2 .

2. L'idéal $\mathcal{P}(a, b)$ est $\mathfrak{D}^{*}$-stable si et seulement si $(a, b) \in \mathcal{E}$.

3. Si $(a, b) \notin \mathcal{E}$, alors l'idéal engendré par $\mathcal{P}(a, b)$ et $\mathfrak{D}^{*} \mathcal{P}(a, b)$ a hauteur $\geq 3$.

Esquisse de démonstration. (1). On vérifie que le radical de $\mathcal{P}(a, b)$ dans $\mathcal{T}^{*}$ pour $a, b \in \mathbb{C}^{\times}$est l'idéal premier de hauteur 2 :

$$
\left(b \varphi_{2}^{3}-\chi_{6}, a \varphi_{2}^{2} \chi_{6}-b \chi_{5}^{2}, b^{2} \varphi_{2} \chi_{5}^{2}-a \chi_{6}^{2}\right) .
$$

Si $a=b=0$, le radical de $\mathcal{P}(0,0)$ est l'idéal $\left(\chi_{5}, \chi_{6}\right)$.

(2). Nous voulons trouver tous les couples $(a, b) \in \mathbb{C}^{2}$ tels que

$$
d^{*}\left(a \varphi_{2}^{5}-\chi_{5}^{2}\right), d^{*}\left(b \varphi_{2}^{3}-\chi_{6}\right) \in \mathcal{P}(a, b) .
$$

On obtient, à l'aide de la proposition 3 :

$$
\begin{aligned}
d^{*}\left(a \varphi_{2}^{5}-\chi_{5}^{2}\right)= & -3 \varphi_{2}^{2} \chi_{6}\left(a \varphi_{2}^{5}-\chi_{5}^{2}\right)+ \\
& \frac{7}{5} \varphi_{2} \chi_{6}\left(\chi_{6}+\varphi_{2}^{3}(3000 a+b)\right)\left(b \varphi_{2}^{3}-\chi_{6}\right)- \\
& \frac{7}{5} \varphi_{2}^{7} \chi_{6}\left(b^{2}+3000 a b-5 a\right), \\
d^{*}\left(b \varphi_{2}^{3}-\chi_{6}\right)= & -350 \chi_{5}\left(a \varphi_{2}^{5}-\chi_{5}^{2}\right)+ \\
& \frac{2}{5} \varphi_{2}^{2} \chi_{5}(6300 b-1)\left(b \varphi_{2}^{3}-\chi_{6}\right)+ \\
& \frac{14}{5} \varphi_{2}^{5} \chi_{5}\left(125 a+b-900 b^{2}\right) .
\end{aligned}
$$

Pour que $d^{*}\left(a \varphi_{2}^{5}-\chi_{5}^{2}\right), d^{*}\left(b \varphi_{2}^{3}-\chi_{6}\right) \in \mathcal{P}(a, b)$, il faut et il suffit que $125 a+$ $b-900 b^{2}=b^{2}+3000 a b-5 a=0$, c'est-à-dire, que $(a, b)$ appartienne à $\mathcal{E}$. Sur la base de ces informations, on vérifie ensuite que les idéaux $\mathcal{P}(0,0), \mathcal{P}(1 / 800000$, 
$1 / 800), \mathcal{P}(1 / 253125,1 / 675)$ sont aussi $\mathfrak{D}^{*}$-stables, et que les idéaux $\mathcal{Q}(0,0)$, $\mathcal{Q}(1 / 800000,1 / 800), \mathcal{Q}(1 / 253125,1 / 675)$ sont $\mathfrak{D}$-stables.

(3). On utilise, comme pour la propriété (2), la proposition 3. On démontre en calculant des résultants, que si $\mathcal{P}(a, b)$ n'est pas $\mathfrak{D}^{*}$-stable, alors l'idéal engendré par $\mathcal{P}(a, b)$ et $\mathfrak{D}^{*} \mathcal{P}(a, b)$ contient les trois éléments $\varphi_{2}, \chi_{5}, \chi_{6}$.

Démonstration de la proposition 3. On vérifie que si $(a, b) \in \mathcal{E}$, alors $\mathcal{Q}(a, b)$ a hauteur 2 car $\chi_{15}^{2} \in \mathcal{P}(a, b)$.

Soit $\mathcal{P}$ un idéal premier non nul de hauteur $\geq 2, \mathfrak{D}^{*}$-stable. Suivant la démonstration du lemme 3 , tous les coefficients $\tilde{m}_{i, j}$ de la matrice $\tilde{M}$ appartiennent à $\mathcal{P}$.

La proposition 3 permet de calculer $\tilde{m}_{i, j}$ explicitement. Pour $(i, j),(h, k) \in$ $\{1,2,3\},(i, j) \neq(h, k)$, on calcule ensuite le résultant :

$$
R_{i, j, h, k, \varrho}:=\operatorname{Rés}_{\varrho}\left(\tilde{m}_{i, j}, \tilde{m}_{h, k}\right), \quad \varrho=\chi_{5}, \chi_{6},
$$

qui sont des éléments isobares de $\mathcal{P}$; on vérifie, en utilisant de préference un logiciel de calcul symbolique, que ces éléments sont tous non nuls.

On peut factoriser explicitement ces résultants dans $\overline{\mathbb{Q}}$. Pour des raisons de poids, on trouve que, pour tout $i, j, h, k$ comme ci-dessus :

$$
\begin{aligned}
& R_{i, j, h, k, \chi_{6}}=\lambda \varphi_{2}^{\alpha} \chi_{5}^{\beta} \prod_{s=1}^{t}\left(a_{s} \varphi_{2}^{5}-\chi_{5}^{2}\right) \\
& R_{i, j, h, k, \chi_{5}}=\mu \varphi_{2}^{\alpha^{\prime}} \chi_{6}^{\gamma^{\prime}} \prod_{s=1}^{t^{\prime}}\left(b_{s} \varphi_{2}^{3}-\chi_{6}\right),
\end{aligned}
$$

avec $\alpha, \beta, \ldots, t^{\prime}$ entiers positifs, $\lambda, \mu \in \mathbb{C}^{\times}, a_{s}, b_{s}$ des nombres complexes non nuls, le tout dépendant de $i, j, k, h$.

Les résultants $R_{i, j, h, k, \varrho}$ appartiennent tous à $\mathcal{P}$, et comme $\mathcal{P}$ est premier, au moins un facteur de $R_{i, j, h, k, \chi_{6}}$ à droite de (19) et au moins un facteur de $R_{i, j, h, k, \chi_{5}}$ à droite de $(20)$, se trouvent dans $\mathcal{P}$.

D'après le lemme 4 , nous pouvons supposer que $\varphi_{2}, \chi_{5}, \chi_{6} \notin \mathcal{P}$, car dans ce cas, nous savons déjà que $\mathcal{P}(0,0) \subset \mathcal{P}$.

Donc $\mathcal{P}$ contient un idéal de la forme $\mathcal{P}(a, b)$ avec $a, b$ complexes non nuls. Si la hauteur de $\mathcal{P}$ est 3 , alors $\mathcal{P} \cap\left(\mathbb{C}\left[\varphi_{2}\right] \cup \mathbb{C}\left[\chi_{5}\right] \cup \mathbb{C}\left[\chi_{6}\right]\right) \neq\{0\}$, et $\mathcal{P}(0,0)=\left(\chi_{5}^{2}, \chi_{6}\right) \subset \mathcal{P}$ (lemme 4). Supposons que $\mathcal{P}$ ait hauteur 2. L'idéal $\mathcal{P}(a, b)$ ne pouvant pas être principal, il doit avoir hauteur 2 et doit être $\mathfrak{D}^{*}$-stable, d'après le lemme 9. 
Le lemme 9 implique $(a, b) \in \mathcal{E}$, ce qui prouve la partie de l'enoncé de la proposition 4 concernant l'idéal $\mathcal{P}$.

La propriété concernant les idéaux $\mathfrak{D}$-stables de $\mathcal{T}$, se déduit de ce que nous venons de démontrer, car d'après le lemme $5, \mathcal{T}$ n'a pas d'idéal non nul principal et $\mathfrak{D}$-stable.

\section{Appendice.}

Lemme 10 Soit $F$ une forme quasi-modulaire de Hilbert de poids $\left(k_{1}, \ldots, k_{n}\right)$ et profondeur s. Soit

$$
P=\sum_{s_{1}+\cdots+s_{n} \leq s} f_{s_{1}, \ldots, s_{n}}(z) X_{1}^{s_{1}} \cdots X_{n}^{s_{n}}
$$

le polynôme $P$ associé à $F$ dans (1). Alors $k \geq 2$ s, et pour tout $s_{1}, \ldots, s_{n}$ tel que $s_{1}+\cdots+s_{n}=s$, la fonction $f_{s_{1}, \ldots, s_{n}}(z)$ est une forme modulaire de Hilbert de poids $\left(k_{1}-2 s_{1}, \ldots, k_{n}-2 s_{n}\right)$.

Démonstration. Soient $A, B$ deux éléments de $\Gamma_{K}$ et écrivons :

$$
A=\left(\begin{array}{ll}
a & b \\
c & d
\end{array}\right), \quad B=\left(\begin{array}{ll}
\alpha & \beta \\
\gamma & \delta
\end{array}\right), \quad A B=\left(\begin{array}{ll}
u & v \\
x & y
\end{array}\right),
$$

soit $F$ comme dans les hypothèses du lemme. Nous avons :

$$
\begin{aligned}
F & (A B(z))= \\
= & \prod_{i=1}^{n}\left(c_{i} B_{i}\left(z_{i}\right)+d_{i}\right)^{k_{i}} \sum_{s_{1}+\cdots+s_{n} \leq s} c_{s_{1}, \ldots, s_{n}}(B(z)) \times \\
& \prod_{i=1}^{n}\left(\left(\gamma_{i} z_{i}+\delta_{i}\right)^{2}\left(\frac{x_{i}}{x_{i} z_{i}+y_{i}}-\frac{\gamma_{i}}{\gamma_{i} z_{i}+\delta_{i}}\right)\right)^{s_{i}},
\end{aligned}
$$


donc :

$$
\begin{aligned}
& F(A B(z))= \\
& =\prod_{i=1}^{n}\left(c_{i} B_{i}\left(z_{i}\right)+d_{i}\right)^{k_{i}} \sum_{s_{1}+\cdots+s_{n} \leq s} c_{s_{1}, \ldots, s_{n}}(B(z)) \times \\
& \prod_{i=1}^{n}\left(\gamma_{i} z_{i}+\delta_{i}\right)^{2 s_{i}} \times \\
& \sum_{t_{i}=0}^{s_{i}}\left(\begin{array}{c}
s_{i} \\
t_{i}
\end{array}\right)(-1)^{s_{i}-t_{i}}\left(\frac{x_{i}}{x_{i} z_{i}+y_{i}}\right)^{t_{i}}\left(\frac{\gamma_{i}}{\gamma_{i} z_{i}+\delta_{i}}\right)^{s_{i}-t_{i}} \\
& =\prod_{i=1}^{n}\left(c_{i} B_{i}\left(z_{i}\right)+d_{i}\right)^{k_{i}} \times \\
& \sum_{s_{1}=0}^{s} \sum_{s_{2}=0}^{s-s_{1}} \cdots \sum_{s_{n}=0}^{s-s_{1}-\cdots-s_{n-1}} c_{s_{1}, \ldots, s_{n}}(B(z)) \prod_{i=1}^{n}\left(\gamma_{i} z_{i}+\delta_{i}\right)^{2 s_{i}} \times \\
& \sum_{t_{i}=0}^{s_{i}}\left(\begin{array}{c}
s_{i} \\
t_{i}
\end{array}\right)(-1)^{s_{i}-t_{i}}\left(\frac{x_{i}}{x_{i} z_{i}+y_{i}}\right)^{t_{i}}\left(\frac{\gamma_{i}}{\gamma_{i} z_{i}+\delta_{i}}\right)^{s_{i}-t_{i}} \\
& =\prod_{i=1}^{n}\left(c_{i} B_{i}\left(z_{i}\right)+d_{i}\right)^{k_{i}} \sum_{t_{1}+\cdots+t_{n} \leq s} \prod_{i=1}^{n}\left(\frac{x_{i}}{x_{i} z_{i}+y_{i}}\right)^{t_{i}} \times \\
& \sum_{s_{1}=t_{1}}^{s} \sum_{s_{2}=t_{2}}^{s-s_{1}} \ldots \sum_{s_{n}=t_{n}}^{s-s_{1}-\cdots-s_{n-1}} f_{s_{1}, \ldots, s_{n}}(B(z)) \prod_{i=1}^{n}\left(\gamma_{i} z_{i}+\delta_{i}\right)^{2 s_{i}} \times \\
& \left(\begin{array}{c}
s_{i} \\
t_{i}
\end{array}\right)(-1)^{s_{i}-t_{i}}\left(\frac{\gamma_{i}}{\gamma_{i} z_{i}+\delta_{i}}\right)^{s_{i}-t_{i}} .
\end{aligned}
$$

D'autre part :

$$
\begin{aligned}
& F(A B(z))= \\
& \quad=\prod_{i=1}^{n}\left(x_{i} z_{i}+y_{i}\right)^{k_{i}} \sum_{s_{1}+\cdots+s_{n} \leq s} f_{s_{1}, \ldots, s_{n}}(z) \prod_{i=1}^{n}\left(\frac{x_{i}}{x_{i} z_{i}+y_{i}}\right)^{s_{i}} .
\end{aligned}
$$

Ces deux expressions différentes de $F(A B(z))$ doivent coïncider pour tout $A, B$ et $z$. Nous pouvons fixer pour un instant $B, z$ en laissant varier $A$ de 
telle sorte que l'ensemble :

$$
\left\{\left(\frac{q_{1}}{q_{1} z_{1}+r_{1}}, \ldots, \frac{q_{n}}{q_{n} z_{n}+r_{n}}\right)\right\}
$$

soit Zariski dense dans $\mathcal{H}^{n}$. Comme

$$
\prod_{i=1}^{n}\left(x_{i} z_{i}+y_{i}\right)^{k_{i}}=\prod_{i=1}^{n}\left(\left(c_{i} B_{i}\left(z_{i}\right)+d_{i}\right)\left(\gamma_{i} z_{i}+\delta_{i}\right)\right)^{k_{i}},
$$

nous obtenons l'idéntité formelle :

$$
\begin{aligned}
& \prod_{i=1}^{n}\left(\gamma_{i} z_{i}+\delta_{i}\right)^{k_{i}} \sum_{t_{1}+\cdots+t_{n} \leq s} f_{t_{1}, \ldots, t_{n}}(z) X_{1}^{t_{1}} \cdots X_{n}^{t_{n}}= \\
&=\sum_{t_{1}+\cdots+t_{n} \leq s} X_{1}^{t_{1}} \cdots X_{n}^{t_{n}} \times \\
& \sum_{s_{1}=t_{1}}^{s} \sum_{s_{2}=t_{2}}^{s-s_{1}} \cdots \sum_{s_{n}=t_{n}}^{s-s_{1}-\cdots-s_{n-1}} f_{s_{1}, \ldots, s_{n}}(B(z)) \prod_{i=1}^{n}\left(\gamma_{i} z_{i}+\delta_{i}\right)^{2 s_{i}} \times \\
&=\left(\begin{array}{c}
s_{i} \\
t_{i}
\end{array}\right)(-1)^{s_{i}-t_{i}}\left(\frac{\gamma_{i}}{\gamma_{i} z_{i}+\delta_{i}}\right)^{s_{i}-t_{i}} \\
& \sum_{t_{1}+\cdots+t_{n} \leq s} X_{1}^{t_{1}} \cdots X_{n}^{t_{n}} \times \\
& \sum_{s_{1}=t_{1}}^{s} \sum_{s_{2}=t_{2}}^{s-s_{1}} \sum_{s-s_{1}-\cdots-s_{n-1}}^{n} \sum_{s_{n}=t_{n}}^{n} f_{s_{1}, \ldots, s_{n}}(B(z)) \times \\
& \prod_{i=1}^{n}\left(\begin{array}{l}
s_{i} \\
t_{i}
\end{array}\right)\left(-\gamma_{i}\right)^{s_{i}-t_{i}}\left(\gamma_{i} z_{i}+\delta_{i}\right)^{s_{i}+t_{i}} .
\end{aligned}
$$

En comparant les coefficients des monômes $X_{1}, \ldots, X_{n}$ nous obtenons, pour tout $\left(t_{1}, \ldots, t_{n}\right)$ tel que $t_{1}+\cdots+t_{n} \leq s$ :

$$
\begin{aligned}
f_{t_{1}, \ldots, t_{n}}(z)= & \sum_{s_{1}=t_{1}}^{s} \sum_{s_{2}=t_{2}}^{s-s_{1}} \ldots \sum_{s_{n}=t_{n}}^{s-s_{1}-\cdots-s_{n-1}} f_{s_{1}, \ldots, s_{n}}(B(z)) \times \\
& \prod_{i=1}^{n}\left(\begin{array}{c}
s_{i} \\
t_{i}
\end{array}\right)\left(-\gamma_{i}\right)^{s_{i}-t_{i}}\left(\gamma_{i} z_{i}+\delta_{i}\right)^{s_{i}+t_{i}-k_{i}}
\end{aligned}
$$


Si $\left(t_{1}, \ldots, t_{n}\right)$ est tel que $t_{1}+\cdots+t_{n}=s$ et $f_{t_{1}, \ldots, t_{n}}(z) \neq 0$, alors la somme indéxée par les $s_{1}, \ldots, s_{n}$ à droite de l'égalité (22) n'a qu'un seul terme, avec $s_{i}=t_{i}, i=1, \ldots, n$. Nous obtenons, pour ce terme :

$$
f_{s_{1}, \ldots, s_{n}}(B(z))=\prod_{i=1}^{n}\left(\gamma_{i} z_{i}+\delta_{i}\right)^{k_{i}-2 s_{i}} f_{s_{1}, \ldots, s_{n}}(z),
$$

égalité valide pour tout $B, z$, ce qui implique que $f_{s_{1}, \ldots, s_{n}}(z)$ est une forme modulaire de Hilbert de poids $\left(k_{1}-2 s_{1}, \ldots, k_{n}-2 s_{n}\right)$; la démonstration du lemme est complète.

Comme conséquence de ce qui precède, on voit que si $F$ est une forme quasi-modulaire de Hilbert non constante, de poids $\left(k_{1}, \ldots, k_{n}\right)$, alors $k_{i} \geq 0$ pour tout $i=1, \ldots, n$. De plus, une forme quasi-modulaire de Hilbert de poids $(0, \ldots, 0)$ est une fonction constante.

Lemme 11 Supposons que $[K: \mathbb{Q}]>1$ et soit $F$ une forme quasi-modulaire de Hilbert dont le poids $\left(k_{1}, \ldots, k_{n}\right)$ est non nul et satisfait $\prod_{i=1}^{n} k_{i}=0$ : alors $F=0$.

Démonstration. Supposons par l'absurde qu'il existe une forme quasi-modulaire de Hilbert $F$ non nulle, de poids $\left(k_{1}, \ldots, k_{n}\right)$ et de profondeur $s$, avec la propriété que $k_{j}>0$ et $k_{h}=0$; soit $P$ le polynôme associé (21). Nous allons procéder en deux étapes.

(1). Nous démontrons que $k_{i}$ est pair pour tout $i=1, \ldots, n$, que $\sum_{i} k_{i}=2 s$, et qu'il n'existe qu'un seul $n$-uple d'entiers positifs $\left(s_{1}, \ldots, s_{n}\right)$ avec $\sum_{i} s_{i}=s$ et $f_{\underline{s}} \neq 0$ dans $(21)$. Nous prouvons de plus que $f_{\underline{s}}$ est constante.

Clairement, nous pouvons aussi supposer que $s>0$, car sinon $F$ est une forme modulaire de Hilbert non nulle, en contradiction avec le lemme 6.3 p. 18 de [5].

Le lemme 10 nous dit que $\sum_{i} k_{i} \geq 2 s$ et que pour tout $\left(s_{1}, \ldots, s_{n}\right)$ tel que $\sum_{i} s_{i}=s$ et $f_{\underline{s}} \neq 0$, alors $k_{i} \geq 2 s_{i}$.

Si $s$ satisfait $\sum_{i} k_{i}>2 s$, alors pour tout $\left(s_{1}, \ldots, s_{n}\right)$ tel que $\sum_{i} s_{i}=s$ et $f_{\underline{s}} \neq 0$, il existe $j$ tel que $k_{j}>2 s_{j}$. De plus, par hypothèse, il existe $h$ tel que $k_{h}=0$, ce qui implique $s_{h}=0$. Donc $k_{j}-2 s_{j}>0$ et $k_{h}-2 s_{h}=0$.

Le lemme 10 implique que $f_{s_{1}, \ldots, s_{n}}$ est une forme modulaire de Hilbert de poids $\left(k_{1}-2 s_{1}, \ldots, k_{n}-2 s_{n}\right)$ et le lemme 6.3 p. 18 de [5] implique $f_{s_{1}, \ldots, s_{n}}=0$. Mais alors, dans ce cas, le degré de $P$ est $<s$ d'où une contradiction. 
La seule possibilité est donc que $\sum_{i} k_{i}=2 s, k_{i} \geq 2 s_{i}$ (ce qui implique $k_{i}=2 s_{i}$ pour $i=1, \ldots, n$ et $k_{i}$ pair). Il n'existe qu'un $n$-uple d'entiers positifs $\left(s_{1}, \ldots, s_{n}\right)$ tel que $\sum_{i} s_{i}=s$, et $f_{\underline{s}} \neq 0$. D'après le lemme (10), la forme modulaire de Hilbert $f_{s_{1}, \ldots, s_{n}}$ est de poids $\underline{0}$ et c'est donc une constante non nulle $\lambda \in \mathbb{C}$.

(2). Etant donné un poids $\left(k_{1}, \ldots, k_{n}\right)$ tel que $k_{j}>0$ et $k_{h}=0$, nous démontrons que l'espace vectoriel $\mathcal{Y}(K)_{\left(k_{1}, \ldots, k_{n}\right)}$ a dimension au plus 1 sur $\mathbb{C}$.

En effet, si $F, G \in \mathcal{Y}(K)_{\left(k_{1}, \ldots, k_{n}\right)}$ sont non nulles, alors elles ont profondeur $(1 / 2) \sum_{i} k_{i}$ et

$$
f_{k_{1} / 2, \ldots, k_{n} / 2}(z)=\lambda, \quad g_{k_{1} / 2, \ldots, k_{n} / 2}(z)=\mu,
$$

avec $\lambda, \mu \in \mathbb{C}^{\times}$. Donc :

$$
\mu F-\lambda G \in \mathcal{Y}(K)_{\left(k_{1}, \ldots, k_{n}\right)}
$$

est une forme quasi-modulaire de Hilbert de profondeur $<\sum_{i} k_{i}$, qui est nulle d'après l'étape (1).

Nous terminons la démonstration du lemme : soit $F$ non nul comme dans les hypothèses. Le poids de $F^{3}$ et le poids de $D_{1}^{k_{1}} \cdots D_{n}^{k_{n}} F$ sont égaux à $\left(3 k_{1}, \ldots, 3 k_{n}\right)$ : ces fonctions sont linéairement indépendantes, et il existe un nombre complexe $\tau$ tel que l'équation aux dérivées partielles suivante soit satisfaite :

$$
D_{1}^{k_{1}} \cdots D_{n}^{k_{n}} F=\tau F^{3} .
$$

Mais $F$ admet une expansion en série de Fourier à l'infini; en comparant les coefficients de cette expansion on trouve $F=0$, d'où une contradiction qui termine la preuve du lemme.

Démontrons le théorème 4 . Soit $\mathfrak{E}$ le sous-ensemble de $\mathbb{N}^{n}$ dont les points $\left(k_{1}, \ldots, k_{n}\right)$ sont tels que $\mathcal{Y}(K)_{\left(k_{1}, \ldots, k_{n}\right)} \neq(0)$.

Supposons par l'absurde que $\mathcal{Y}(K)$ soit de type fini, engendré par des élément $F_{1}, \ldots, F_{m}$. On peut supposer que $F_{1}, \ldots, F_{m}$ soient des formes quasimodulaires de Hilbert de poids $\underline{k}_{1}, \ldots, \underline{k}_{m}$. Alors :

$$
\mathfrak{E}=\mathbb{N}_{1}+\cdots+\underline{\mathbb{k}}_{m} .
$$

Soit $\mathfrak{C}$ l'enveloppe convexe de $\mathfrak{E}$ de $\mathbb{R}^{n}$ : alors $\mathfrak{C}=\mathbb{R}_{\geq 0} \underline{k}_{1}+\cdots+\mathbb{R}_{\geq 0} \underline{k}_{m}$ est un cône polyhedral qui ne contient aucun angle plan d'amplitude $\geq \pi / 2$, car d'après le lemme 11 , si $\left(k_{1}, \ldots, k_{n}\right) \in \mathfrak{E}$, alors $\prod_{i} k_{i} \neq 0$. 
Donc $\mathfrak{C}$ n'est pas stable par les translations par les vecteurs de la base canonique de $\mathbb{R}^{n}$. Mais $\mathcal{Y}(K)$ est un anneau différentiel; donc $\mathfrak{E}$ doit être stable par tous les éléménts de $\mathbb{N}^{n}$, et $\mathfrak{C}$ également. Ceci mène à une contradiction, et la preuve du théorème 4 est terminée.

\section{Références}

[1] W. L. Baily, A. Borel. Compactification of arithmetic quotients of bounded symmetric domains. Ann. Math. 84, 442-528 (1966).

[2] J. H. Bruinier, M. Bundschuh. On Borcherds products associated with lattices of prime discriminant. à paraître dans Ramanujan J. (2003).

[3] M. Eichler. Projective varieties and modular forms. Maryland, Printemps 1970. Lecture Notes in Mathematics. 210. Springer-Verlag. (1971).

[4] M. Eichler. On the graded rings of modular forms. Acta Arith. 18, 87-92 (1971).

[5] G. van der Geer. Hilbert modular surfaces. Ergebnisse der Mathematik und ihrer Grenzgebiete. 3. Folge, Bd. 16. Berlin etc. : Springer-Verlag. (1988)

[6] K.-B. Gundlach. Die Bestimmung der Funktionen zur Hilbertschen Modulgruppe des Zahlkörpers $Q(\sqrt{5})$. Math. Ann. 152, 226-256 (1963).

[7] F. Hirzebruch. Hilbert's modular group of the field $\mathbb{Q}(\sqrt{5})$ and the cubic diagonal surface of Clebsch and Klein. Uspekhi Mat. Nauk 31 (1976) 153-166, traduit dans Russian Math. Surveys 31 :5 (1976) 96-110.

[8] M. Kaneko \& D. Zagier. A generalized Jacobi theta function and quasimodular forms. Dijkgraaf, R. H. (ed.) et al., The moduli space of curves. Basel : Birkhäuser. Prog. Math. 129, 165-172 (1995).

[9] Yu. V. Nesterenko. Modular functions and transcendence questions. Sb. Math. 187, 1319-1348 (1996) traduction de Mat. Sb. 187, No.9, 65-96 (1996).

[10] Yu. V. Nesterenko, P. Philippon (éditeurs). Introduction to algebraic independence theory. Lecture Notes in Mathematics. 1752. Berlin : Springer. (2001)

[11] F. Pellarin. Introduction aux formes modulaires de Hilbert et à leur propriétés différentielles. A paraître dans S.M.F., Séminaires et Congrés. 
[12] F. Pellarin. La structure différentielle de l'anneau des formes quasimodulaires pour $\mathbf{S L}_{2}(\mathbf{Z})$. Soumis au Journal de Théorie des Nombres de Bordeaux.

[13] F. Pellarin. Lemmes de multiplicité associés aux groupes triangulaires de Riemann-Schwarz. Soumis à Compositio Math.

[14] H. L. Resnikoff. Automorphic forms and automorphy preserving differential operators on products of halfplanes. Abh. Math. Semin. Univ. Hamburg. 38, 168-198 (1972).

[15] J.-P. Serre. Cours d'arithmétique. Presses Universitaires de France, quatrième édition (1994).

[16] D. Zagier. Modular forms and differential operators. Proc. Indian Acad. Sci. (Math. Sci.) Vol. 104, No. 1, pp. 57-75 (1994). 